\title{
Examining the 'Copy and Paste' Function in the Use of Electronic Health Records
}

\author{
Svetlana Z. Lowry \\ Mala Ramaiah \\ Sandra Spickard Prettyman \\ Debora Simmons \\ David Brick \\ Ellen Deutsch \\ Lorraine Possanza \\ Ollie B. Gray \\ Betty A. Levine \\ Kinsey Gimbel \\ Kyle Andrews
}

This publication is available free of charge from:

https://doi.org/10.6028/NIST.IR.8166 


\section{Examining the 'Copy and Paste' Function in the Use of Electronic Health Records}

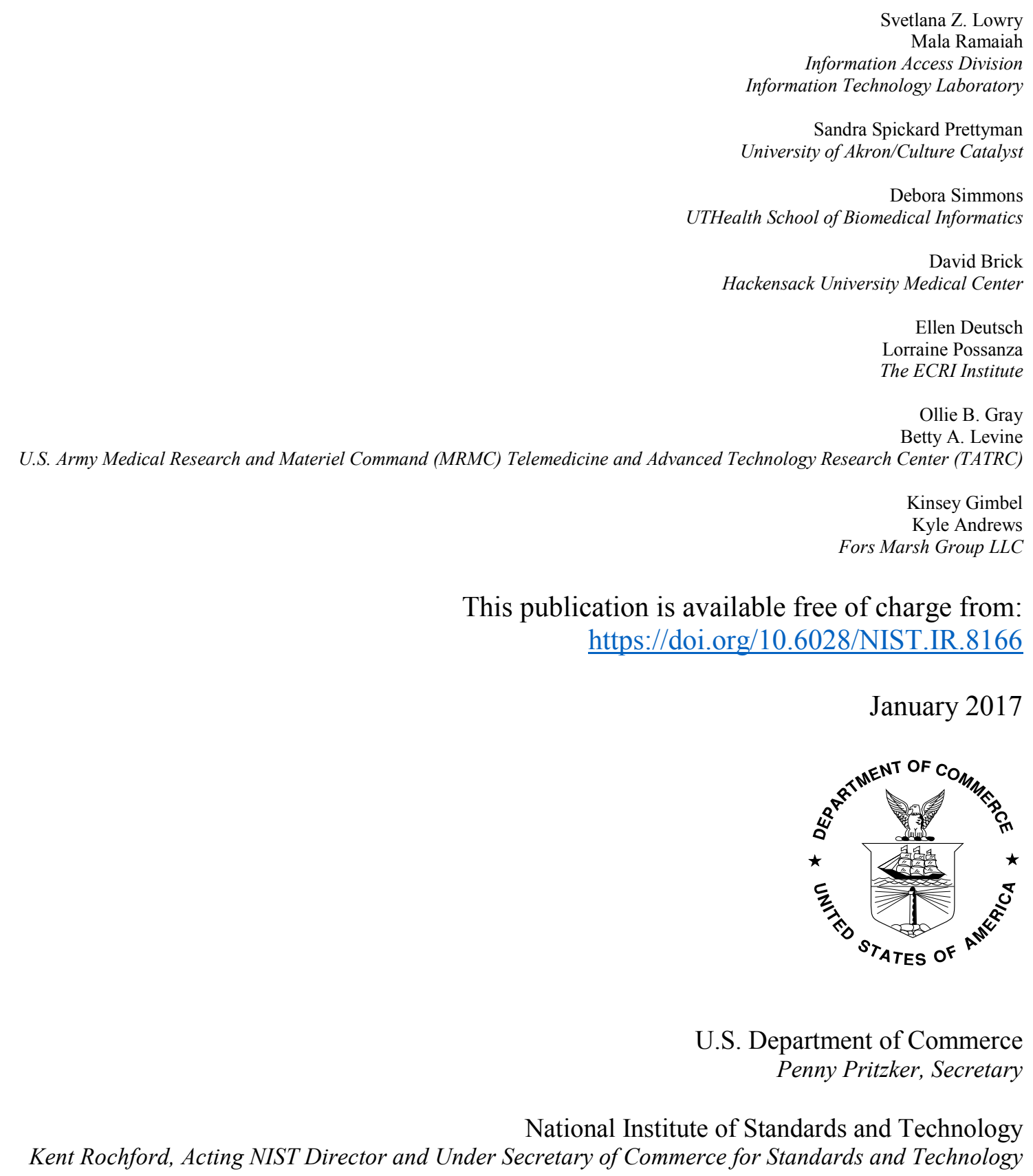




\section{Acknowledgements}

Adam Bazer, Senior Manager, Health Information Systems, HIMSS North America

Gerard M. Castro, PhD, MPH, Project Director, Patient Safety Initiatives, Office of Patient Safety, The Joint Commission

Jorge Ferrer, MD, MBA, LSA, Health Informatician, Veterans Health Administration and Adjunct Assistant Professor, UTHealth School of Biomedical Informatics

Tejal Gandhi, MD, MPH, CPPS, President \& CEO, National Patient Safety Foundation Amy Goldberg-Alberts, MBA, FASHRM, CPHRM, Executive Director, Partnership Solutions, Patient Safety, Risk, and Quality, ECRI Institute

Brian Griepentrog, PhD, Senior Vice President, Fors Marsh Group

Paul Latkany, MD, New York Eye and Ear Infirmary of Mt. Sinai

Joe Lewelling, Vice President, Emerging Technologies and Health IT, Association for the Advancement of Medical Instrumentation

Edwin A. Lomotan, MD, FAAP, Chief of Clinical Informatics, Health Information Technology Division, Center for Evidence and Practice Improvement, Agency for Healthcare Research and Quality

Larry Nadel, PhD, Electronics Engineer, Information Access Division, National Institute of Standards and Technology

Anna Orlova, PhD, Senior Director, Standards, American Health Information Management Association

Raj Ratwani, PhD, Scientific Director, National Center for Human Factors in Healthcare, MedStar

Jeanie Scott, MS, CPHIMS, Director, Informatics Patient Safety, Veterans Health Administration

Ronni Solomon, JD, Executive Vice President and General Counsel, ECRI Institute

Sheryl Taylor, BSN, RN, IT Specialist, Software and Systems Division, National Institute of Standards and Technology 


\section{DISCLAIMER}

Certain commercial entities, equipment, or material may be identified in this document in order to describe a concept adequately. Such identification is not intended to imply recommendation or endorsement by the National Institute of Standards and Technology, nor is it intended to imply that these entities, materials, or equipment are necessarily the best available for the purpose. 


\section{Contents}

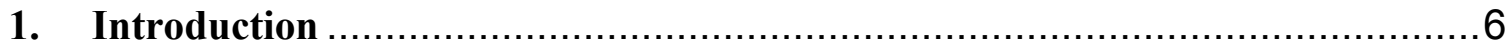

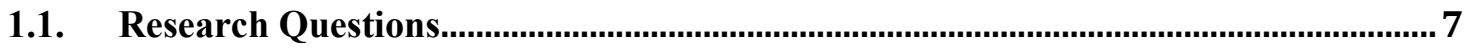

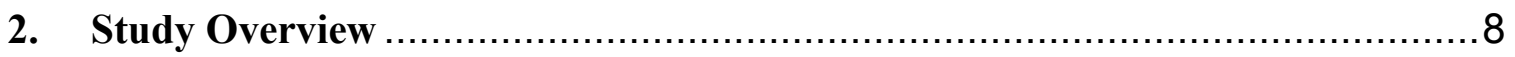

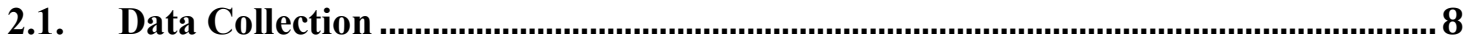

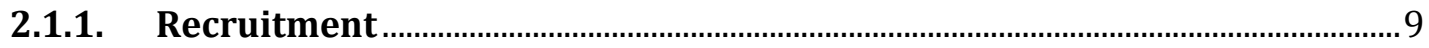

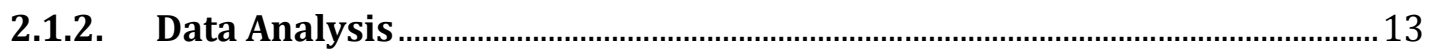

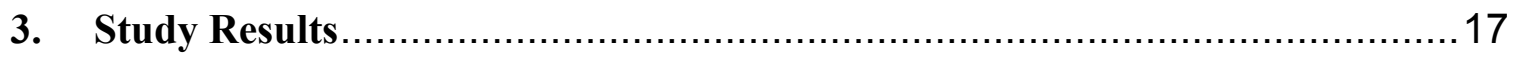

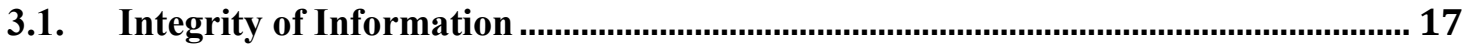

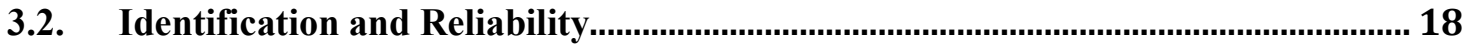

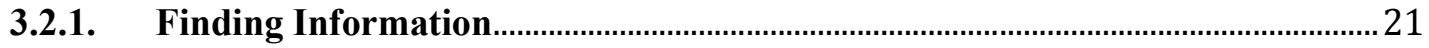

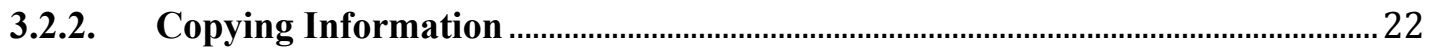

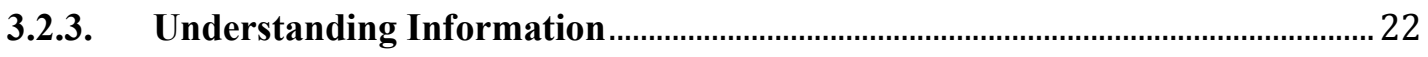

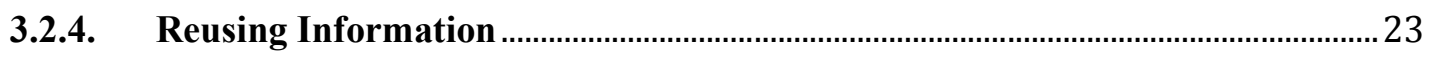

4. Empirical Evidence to the Partnership Recommendations on 'copy and paste'

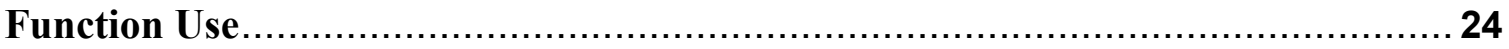

4.1. Recommendation A: Provide a Mechanism to Make 'copy and paste' Material

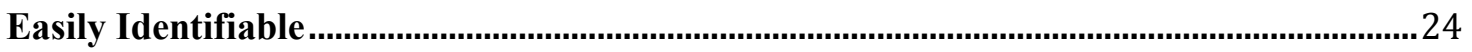

4.2. Recommendation B: Ensure That the Provenance of 'copy and paste' Material Is

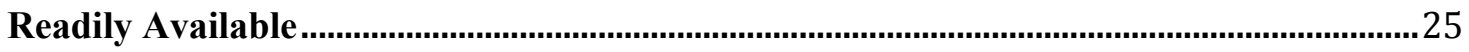

4.3. Recommendation C: Ensure adequate staff training and education regarding the

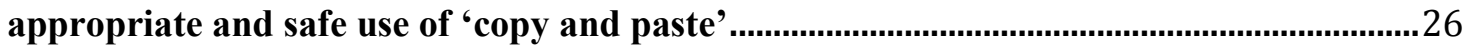

5. Human Factors Assessments of the 'copy and paste' Function ..................27

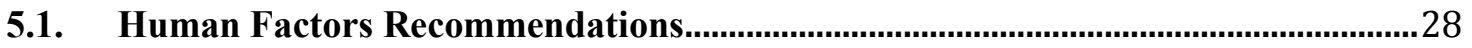




\subsubsection{Human Factors Recommendations to support The Partnership}

Recommendation A: Provide a mechanism to make 'copy and paste' material easily identifiable

\subsubsection{Human Factors Recommendation to support The Partnership}

Recommendation B: Ensure that the provenance of 'copy and paste' material is readily available

\subsubsection{Additional Human Factors Recommendations for critical areas identified in}

the study

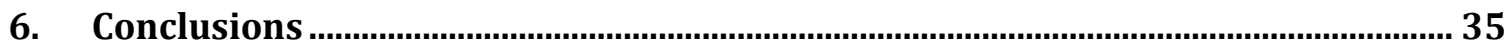

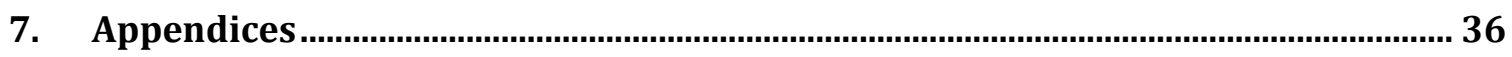

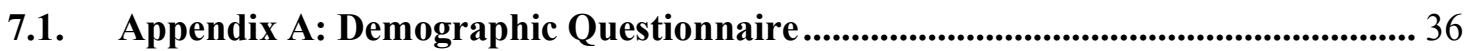

7.2. Appendix B: Post-Task And Post-Test Questions ....................................................... 38

7.3. Appendix C: Semistructured In-depth Interview.......................................................... 39

7.4. Appendix D: Testing Scenarios and Tasks............................................................... 42 


\section{Executive Summary}

In 2015, the National Institute of Standards and Technology (NIST) released NISTIR 7804-1, Technical Evaluation, Testing, and Validation of the Usability of Electronic Health Records: Empirically Based Use Cases for Validating Safety-Enhanced Usability and Guidelines for Standardization ${ }^{1}$. That research established an empirical rationale for critical patient safety-related usability guidelines for standardization. It also showed that the integrity of information in Electronic Health Records (EHRs) is frequently compromised by how data is used and reused, one among other findings. One of the major contributors to this lack of integrity of information is the 'copy and paste' functionality which enables information to be rapidly and readily reused.

This paper describes the findings obtained from a usability study of the 'copy and paste' functionality in EHRs. 'Copy and paste' functionality is intended to allow medical practitioners to easily and efficiently reuse information in patient EHRs without having to retype the information. However, in practice, current implementation of this functionality has introduced overwhelming and unintended safety-related issues into the clinical environment.

The copied and pasted text may look like this text in the box below:

"This is how copied and pasted text could appear in the clinical records. This is how copied and pasted text could appear in the clinical records. This is how This is how This is how copied and pasted text could appear in the clinical records. The patient is allergic to penicillin. This is how copied and pasted text could appear in the clinical

This is how copied and pasted text could

appear in the clinical records. The patient is allergic to penicillin. This is how copied and pasted text could appear in the clinical records.

This is how copied and pasted text could appear in the clinical records
This is how copied and pasted text could appear in the This is how copied and pasted text could appear in the clinical records. This is how copied and pasted text could appear in the clinical records

This is how copied and pasted text could appear in the clinical records. This is how copied and pasted text could appear in the clinical records. The patient is allergic to penicillin. This is how copied and pasted text could appear in the clinical records. The patient is allergic to penicillin. The patient is allergic to penicillin. The patient is allergic to penicillin. The patient is allergic to penicillin. The patient is allergic to penicillin."

${ }^{1}$ Lowry, S.Z., et al. (2015). Technical Evaluation, Testing, and Validation of the Usability of Electronic Health Records: Empirically Based Use Cases for Validating Safety-Enhanced Usability and Guidelines for Standardization NISTIR 7804-1. 
The Partnership for Health IT Patient Safety convened by ECRI Institute released a special report Copy/Paste: Prevalence, Problems, and Best Practices ${ }^{2}$. Subsequently, the Partnership released recommendations for the safe use of 'copy and paste' function.

The four ECRI recommendations are:

Recommendation A: Provide a mechanism to make 'copy and paste' material easily identifiable.

Recommendation B: Ensure that the provenance of 'copy and paste' material is readily available.

Recommendation $\mathrm{C}$ : Ensure adequate staff training and education regarding the appropriate and safe use of 'copy and paste'.

Recommendation D: Ensure that 'copy and paste' practices are regularly monitored, measured, and assessed.

The National Institute of Standards and Technology (NIST), in collaboration with ECRI and U.S. Army Medical Research and Materiel Command's (MRMC) Telemedicine and Advanced Technology Research Center (TATRC), conducted this study, which was intended to help fill the gap in understanding the implications of the 'copy and paste' functionality by providing a more in-depth examination of the 'copy and paste' function in EHRs as to how the practitioners utilize it, exploring if and when they use it. As well as, their perception about it and the ways in which it may or may not lead to errors that could affect patient safety. Finally, substantiating the ECRI Institute convened Partnership for Health IT Patient Safety's recommendations for the safe use of 'copy and paste' function in health IT by providing human factors recommendations for the safe use of the 'copy and paste' function based on the study findings and the preceding analysis. The Partnership focused on the issue of 'copy and paste' function because it was known that the process was widely used but the issue of the consequences was not fully recognized. It was warranted to have a small sample size to focus on the recommendations, given the more extensive analysis of the prior reported events that were studied by The Partnership in conjunction with the information obtained in the evidence-based literature review. Therefore, this sample served only as validation of the preceding analysis.

Data collection took place from July-August, 2016, at the Fors Marsh Group facility in the Washington, D.C. metropolitan area. Data was collected by a team of researchers observing and interviewing the study participants (physicians and nurses) interacting with an EHR and performing their routine tasks. The study participants accomplished their tasks using the AHLTA Electronic Health Records application hosted in TATRC ${ }^{2}$, Early Stage Platform (ESP).

The goal was to elicit information from the study participants about the use of the 'copy and paste' function and possible patient safety-related issues associated with its use. Another purpose was to examine whether or not and the ways in which the participants' beliefs and experiences in using the 'copy and paste' functionality to document information in the EHRs supported the four safe practice recommendations developed by the Partnership for Health IT Patient Safety convened by the ECRI Institute.

This study explored the ways in which clinical providers (nurses and physicians) use EHRs in their work, specifically, the 'copy and paste' function within the EHRs, if available. The major findings of the usability study are:

2 (2015). Copy/Paste: Prevalence, Problems, and Best Practices. Health Technology Assessment Information Service Special Report. https://www.ecri.org/Resources/HIT/HTAIS_Copy_Paste_Report.pdf

2 http://www.tatrc.org/www/labs-and-programs/health-it/ 
1. Study participants expressed concerns about the integrity of information while using the 'copy and paste' function.

2. Study participants identified a high potential risk of entering wrong information in the wrong chart.

3. Study participants revealed critical issues regarding the 'copy and paste' function in producing "hyper-documentation" or "note bloat," which makes it difficult to extract accurate, relevant, and timely information on a patient.

Additional findings resulting from a human factors assessment of the empirical data obtained from this study of the 'copy and paste' function are identified as follows:

- Amount of information: Unconstrained ability to extract a large amount of information using 'copy and paste' function can present users with convoluted and/or irrelevant information impairing situation awareness. Users may miss important pieces of information.

- Attribution of information: Clinicians need to know the source of the copied and pasted information, who copied and pasted it, what was added to/edited in the information, and the date and time the information was copied and pasted.

- Wrong information: A common error in the use of 'copy and paste' function is that users forget to properly review and edit all of the information they have copied and pasted (frequent interruptions in the clinical environment being one of the major causes of this error) and users are not provided with system features that promote efficient editing.

This report provides specific recommendations for user interface design to improve safety-related usability for the 'copy and paste' function. In summary, these recommendations include:

\section{A. Human Factors Recommendations to support The Partnership Recommendation A: Provide a mechanism to make 'copy and paste' material easily identifiable.}

- EHR systems must be designed to enhance the visibility of the information being selected for 'copy and paste' to prevent users from inadvertently copying only part of the information that was intended to be copied which could minimize the possibility of incomplete reuse of information that could lead to morbid/mortal errors.

- EHR systems should provide a concept for reconciling that the copied information was read consciously and edited by the clinical provider which would promote the attribution of the source of the information.

- In the course of empirical study, there were identified areas that must be locked from copying:

○ The 'copy and paste' function must not be allowed when entering any information into a blood bank information system due to the extreme risk involved in blood transfusion.

- Demographic information: Demographic information should never be copied, but needs to be autopopulated in all the interfaces within a patient's chart.

- Copying of the demographic data from one chart to another should NOT be allowed. NIST research revealed a clear trend in the users' perception that each subsection of the patient chart needs to be autopopulated with the patient demographics. However, there were instances mentioned where manual entry of the demographic data becomes necessary during documentation outside of the EHR platform. Necessary provisions need to be provided in these instances to ensure correct entry of the demographic information.

○ Dates should never be copied and pasted. 
B. Human Factors Recommendation to support The Partnership Recommendation B: Ensure that the provenance of 'copy and paste' material is readily available.

- User interface must display the "chain of custody" of the information associated with the use of “'copy and paste'.' However, this information should not be displayed by default, and be shown only on user demand to avoid the possibility of overwhelming clinical users and contribute to errors of commission (taking an incorrect action).

\section{Additional Human Factors Recommendations for critical areas identified in the study}

- Vital Signs

○ The following properties should be displayed when documenting vital signs through charting done by the clinical user or by real time feed to the EHR system from digital devices:

- Time stamp when measured. How it was measured (nurse manually takes blood pressure, arterial line captures BP at a single time)

- Time stamp when documented in the EHR.

- Time stamp when it is signed (locked).

- Time stamp when it is revised.

- Time stamp when retrieved.

- Allergies

○ There should be clear "chain of custody" when copying and pasting information related to allergies.

- Surgical notes

- The design of the EHR must ensure that all of the information pertaining to surgical notes is provided with the 'copy and paste' functionality which helps the reuse of information by the clinical staff in the "exact" and precise context of the surgical notes. Variability may exist due to patient-specific findings and/or specific situations that might arise unexpectedly during the procedure which must be accommodated by EHRs by means of provisions for efficient editing.

- Medication entry

- When medication entry is done in free text form, the 'copy and paste' function can be the superior method of entry as compared to typing. Copying and pasting a medication with the dosing is much safer compared to the use of a drop down menu since there are known errors related to wrong selections and jumping menus in the use of drop down menus.

- The 'copy and paste' function should always be associated with a clear "chain of custody." As a general rule, the use of 'copy and paste' should be discouraged in new orders to reinforce the fact of conscious data entry.

- Discharge summary

- When used during patient discharge, the 'copy and paste' of reused information should reflect the "chain of custody" for the clinical provider indicating that it is indeed copied from a past event.

- A discharge summary is a good example of a location where 'copy and paste' functionality increases efficiency and efficacy, so long as the clinical provider is presented the "chain of custody" of such information. 
- Copying and pasting information from different departments and another patient's EHR: When copying and pasting information into an EHR from a different patient's chart is allowed, the EHR system must keep the clinician oriented as to which patient's record they are accessing at any given point in the process.

○ There should be a clear "chain of custody" indicating the exact source of the copied and pasted information while protecting the privacy and confidentiality of the source patient.

- Enable user to easily transition from the current chart with unrestricted access to input information to another chart by a deliberate action (i.e., identification / activation of the patient chart) by the user.

The study conclusions include:

The human factors recommendations provided above complement the ECRI Institute convened Partnership for Health IT Patient Safety's recommendations for safe use of “copy and paste'.' They add empirical evidence and human factors methodology to the safe use of 'copy and paste' emphasized by the Partnership recommendations.

Data from this study overwhelmingly support Recommendation A and Recommendation B. There is no direct data to report on Recommendation $\mathrm{C}$ and Recommendation $\mathrm{D}$, although there are some general indicators pointing toward ways in which the participants tangentially provided support for Recommendation $\mathrm{C}$ related to training. 


\section{Introduction}

Currently, in the United States, there is widespread use of Electronic Health Record (EHR) systems. The Health Information Technology for Economic and Clinical Health (HITECH) Act was enacted by the U.S. Congress in 2009. There has been a remarkable increase in the use of these systems since then. The use of EHRs has drastically changed the ways in which patient information is collected, stored, and accessed. In 2015, the National Institute of Standards and Technology (NIST) released NISTIR 7804-1, Technical Evaluation, Testing, and Validation of the Usability of Electronic Health Records: Empirically Based Use Cases for Validating Safety-Enhanced Usability and Guidelines for Standardization $^{3}$. This research established empirical rationale for critical patient safety-related usability guidelines for standardization which showed that the integrity of information in EHRs is frequently compromised by how data is used and reused, one among the other findings. One of the major contributors to this lack of integrity is the 'copy and paste' functionality which enables information to be rapidly and readily reused.

The 'copy and paste' function allows practitioners to reuse information in the patient EHRs, easily and efficiently without having to retype it. Many systems also support a carry/copy function, which is different from the 'copy and paste' function. The carry/copy forward function brings sets of information into a similar area while 'copy and paste' function lets the user to place the information anywhere. Healthcare practitioners facing time constraints during their work day often, prefer to utilize 'copy and paste' function and the carry forward function in order to improve the efficiency and effectiveness with which they can reuse information within the EHR system during clinical documentation. The 'copy and paste' function and carry forward function are considered well-suited for clinical documentation when the end-user considers the information to be "standardized" (e.g., documenting of the steps in a surgical procedure) or similar (e.g., a patient's past clinical history that has not changed from the previous visit).

While it is assumed that there is widespread use of the 'copy and paste' functionality in EHRs, there is a gap in our knowledge and understanding about the use of the 'copy and paste' function in clinical settings during clinical documentation using EHRs; the ways in which the use of the 'copy and paste' function may contribute to errors; and how those errors could impact patient safety and care. In October 2015, the Partnership for Health IT Patient Safety convened by ECRI Institute released a special report Copy/Paste: Prevalence, Problems, and Best Practices ${ }^{4}$. This comprehensive ECRI report provided evidence demonstrating that practitioners routinely use the 'copy and paste' function in their practice. Subsequently, in February 2016, the Partnership released recommendations for the safe use of 'copy and paste' and an accompanying implementation Toolkit for the safe use of 'copy and paste' in their report, Health IT Safe Practices: Toolkit for the Safe Use of 'copy and paste's. The four recommendations in this report are:

Recommendation A: Provide a mechanism to make 'copy and paste' material easily identifiable.

\footnotetext{
${ }^{3}$ Lowry, S.Z., et. al. (2015). Technical Evaluation, Testing, and Validation of the Usability of Electronic Health Records: Empirically Based Use Cases for Validating Safety-Enhanced Usability and Guidelines for Standardization NISTIR 7804-1. ${ }^{4}$ (2015). Copy/Paste: Prevalence, Problems, and Best Practices. Health Technology Assessment Information Service Special Report. https://www.ecri.org/Resources/HIT/HTAIS Copy Paste Report.pdf 5 (2016). Health IT Safe Practices: Toolkit for the Safe Use of 'copy and paste'. ECRI Partnership for Health IT Patient Safety. Partnership for Health IT Patient Safety Report. https://www.ecri.org/Resources/HIT/CP Toolkit/Toolkit_CopyPaste_final.pdf
} 
Recommendation B: Ensure that the provenance of 'copy and paste' material is readily available.

Recommendation C: Ensure adequate staff training and education regarding the appropriate and safe use of 'copy and paste'.

Recommendation D: Ensure that 'copy and paste' practices are regularly monitored, measured, and assessed.

These recommendations and toolkit were the result of a private sector multi-stakeholder health IT initiative that was convened to improve the safety of health information technology, to encourage the safe use of health IT, and to better use health IT safely.

This paper documents the NIST study "Examining the 'copy and paste' Function in the Use of Electronic Health Records" which was intended to help fill the gap in understanding the implications of the 'copy and paste' functionality by providing a more in-depth examination of the 'copy and paste' function in EHRs as to how the practitioners utilize it, exploring if and when they use it. As well as, their perception about it and the ways in which it may or may not lead to errors that could affect patient safety. Finally, substantiating the ECRI Institute convened Partnership for Health IT Patient Safety's recommendations ${ }^{3}$ for the safe use of 'copy and paste' function in health IT by providing human factors recommendations for the safe use of the 'copy and paste' function based on the study findings and the preceding analysis. The Partnership focused on the issue of 'copy and paste' because it was known that the process was widely used but the issue of the consequences was not fully recognized. It was warranted to have a small sample size to focus on the recommendations, given the more extensive analysis of the prior reported events that were studied by The Partnership in conjunction with the information obtained in the evidence-based literature review. Therefore, this sample served only as substantiation of the preceding analysis

\subsection{Research Questions}

The questions below served as guides in the research design for the study and in the development of data collection tools and data analysis processes. They were developed in conjunction with researchers at NIST, the Fors Marsh Group (FMG), the ECRI Institute, and the U.S. Army Medical Research and Materiel Command's (MRMC) Telemedicine and Advanced Technology Research Center (TATRC).

1. In what ways, if at all, do participants (clinicians including physicians and nurses) believe the 'copy and paste' function in EHRs contributes to or helps prevent errors related to patient safety?

2. Will or do the ECRI Institute convened Partnership for Health IT Patient Safety's safe practice recommendations for the use of 'copy and paste' improve/impact/mitigate errors that impair or endanger patient safety?

These questions provided focus for the study and guided the data collection and analysis.

3 (2016). Health IT Safe Practices: Toolkit for the Safe Use of 'copy and paste'. Partnership for Health IT Patient Safety. ECRI Partnership for Health IT Patient Safety. Partnership for Health IT Patient Safety Report. https://www.ecri.org/Resources/HIT/CP Toolkit/Toolkit_CopyPaste final.pdf 


\section{Study Overview}

This NIST study explored the ways in which clinical providers (nurses and physicians) use EHRs in their work, and specifically, the 'copy and paste' function, if available. The goal was to elicit information about the use of 'copy and paste' function and possible patient safety-related issues associated with that use. Another purpose was to examine whether or not and in what ways, participants' beliefs and experiences in using the 'copy and paste' functionality in the EHRs to document information that supported the four safe practice recommendations developed by the ECRI Institute convened Partnership for Health IT Patient Safety.

\subsection{Data Collection}

Data collection took place from July_August, 2016, at the Fors Marsh Group facility in the Washington, D.C. metropolitan area. Data was collected by a team of researchers observing the study participants interacting with an EHR and performing their routine tasks. Follow-up interviews were conducted by the lead qualitative researcher.

The study participants accomplished their tasks using the AHLTA Electronic Health Records application hosted in the TATRC's Early Stage Platform (ESP).

The system was configured by the team from TATRC's Health Technology Innovation Center (HTIC). Researchers were provided access to the test instance of AHLTA that was prepopulated with "synthetic" patient data (generated by a computer).

Researchers explored the 'copy and paste' functionality within the ESP's AHLTA instance. Clinical test scenarios were created using the synthetic data within the ESP's AHLTA instance to reflect the test participants' routine tasks and daily interactions with an EHR to the extent possible, given the testing environment. Certain features of the EHR, such as medication ordering, signing off and accessing radiology reports were not included in the test scenarios due to limitations in the functionality of the AHLTA instance. The participants were not informed that the purpose of the study was to learn about their 'copy and paste' practices and perceptions. They were informed that the general purpose of the research was to learn about EHR users' experiences with system usability. Participants were instructed to perform typical clinical tasks interacting with an EHR system. In the follow-up interviews they were asked to give their feedback and describe their experiences using the EHR. Although the participants were not directly informed about the 'copy and paste' research purpose, significant intentional efforts were made to create clinical test scenarios that "encouraged" potential use of the 'copy and paste' function.

The observations and interview protocol included six parts as outlined below (see Appendix A for a copy of the full protocol).

1. An overview of consent procedure and study process;

2. A demographic questionnaire which included general questions (e.g., age range, educational background, proficiency level using technology and frequency of technology usage);

3. Pre-task questions that focused on current roles and responsibilities and general EHR usage; 
4. An observations section that included a training video for the sample EHR system, a printed material containing the clinical scenario accompanied by the tasks they were supposed to accomplish using the EHR and commands for the participants to read. This was followed by the participants attempting/accomplishing the tasks given to them using the sample EHR system (during which they were observed). Later the participants were asked a series of post-task questions to rate the difficulty level of each task and how likely they thought it would contribute to an error;

5. A post-task debriefing that asked them about performing the tasks and using the sample EHR system. The participants were urged to talk about their use of the 'copy and paste' function. Once this information was elicited, in-depth information about the participant's experiences and perception about 'copy and paste' function was collected; and

6. Final section that included general questions about EHRs as well as specific questions about the use of the 'copy and paste' function in their daily routine tasks.

The scenarios used for the observations segment were developed by the clinical subject matter experts and members of the research team. No actual real patient records were used in the study. All patient records were computer-generated. Sessions lasted between one and two hours. The sessions were video and audio recorded and verbatim transcriptions were made of all interviews. In addition, clinical researchers took observation notes of the participants carrying out the tasks using the EHR. Screen captures reflecting use of the sample EHR system served as supplemental data.

The team of researchers that designed and conducted the study comprised technical, clinical, human factors, usability, safety and informatics experts.

\subsubsection{Recruitment}

Recruitment for the project was conducted by the Fors Marsh Group. Inclusion criteria for participants were that they must be at least 18 years of age, be a male or female clinical provider (physician or nurse) using EHR system/s, and with varying levels of experience using the EHR system. Every effort was made to recruit participants with varying levels of experience working with an EHR system.

\subsubsection{Participant Demographics}

A total of nine clinical healthcare providers participated in the study, including five nurses and four physicians. Participants referred to as $\mathrm{P} 101$ to $\mathrm{P} 104$ were physicians and participants referred to as P201 to P205 were nurses.

\subsubsection{Age, Gender, Education and Experience}

Demographics for both categories (nurses and physicians) are provided below in Table 1. 


\begin{tabular}{|c|c|c|c|c|}
\hline Participant code & Age Range & Gender & Education & $\begin{array}{c}\text { Time in Current } \\
\text { Occupation }\end{array}$ \\
\hline P101 & $55-64$ & M & Graduate Degree & 15 years \\
\hline P102 & $55-64$ & M & Graduate Degree & 4 years \\
\hline P103 & $25-34$ & $\mathrm{~F}$ & Graduate Degree & 5 years \\
\hline P104 & $25-34$ & M & Graduate Degree & 1 year \\
\hline P201 & $18-24$ & $\mathrm{~F}$ & Associate Degree & 2 years \\
\hline P202 & $35-44$ & $\mathrm{~F}$ & Graduate Degree & 3 years \\
\hline P203 & $25-34$ & $\mathrm{~F}$ & $\begin{array}{c}\text { Undergraduate } \\
\text { Degree }\end{array}$ & 3 years \\
\hline P204 & $25-34$ & $\mathrm{~F}$ & $\begin{array}{c}\text { Undergraduate } \\
\text { Degree }\end{array}$ & 1 year \\
\hline P205 & $35-44$ & $\mathrm{~F}$ & Graduate Degree & 5 years \\
\hline
\end{tabular}

Table 1: Participant Demographics

All of the nurses fell within the first three age range categories, and none of the nurses were older than 44. There was a large gap in the physician ages, with two of the physicians in the $25-34$ age category and two who were between 55 and 64 years of age. All of the nurses were female; three of the physicians were male and one was female. Two of the nurses had an undergraduate degree, one held an associate's degree, and two had a graduate degree. All four of the physicians had graduate degrees and two of them held special certifications. All of the nurses self-identified as registered nurses and all of the physicians self-identified as medical doctors (MDs). Nurses had been working in their current positions for between one and five years, and physicians had been working in their current positions for between one and 15 years.

\subsubsection{Technology Proficiency and Use}

The demographic questionnaire asked participants to rate their proficiency level using computers at work and at home, along with frequency of use. It also asked specifically about use of EHRs and the environments in which they were used. One of the physicians had been working with his/her current EHR system for less than six months; two nurses and one physician had been working with their current EHR for more than one year, but less than two years; one physician and three nurses had been working with their current system for more than two years, but less than three years; and one physician had been working with his/her current system for more than three years. Physicians noted that they had worked with two to six different EHR systems over the course of their careers, while nurses reported working with one to five different systems.

Tables 2 and 3 show participant proficiency levels using computers, both at home and work environments and the frequency of this use, respectively. Four out of five nurses and all four physicians reported a "high" proficiency level while one nurse reported medium (relatively 
comfortable) proficiency level using computers. Three nurses and three physicians said they used computers outside of work often (several times a day), while one nurse and one physician said they used computers outside of work frequently (several times a week) and one nurse said she used computers outside of work infrequently (several times a month). All five nurses and four physicians reported using computers at work often (several times a day).

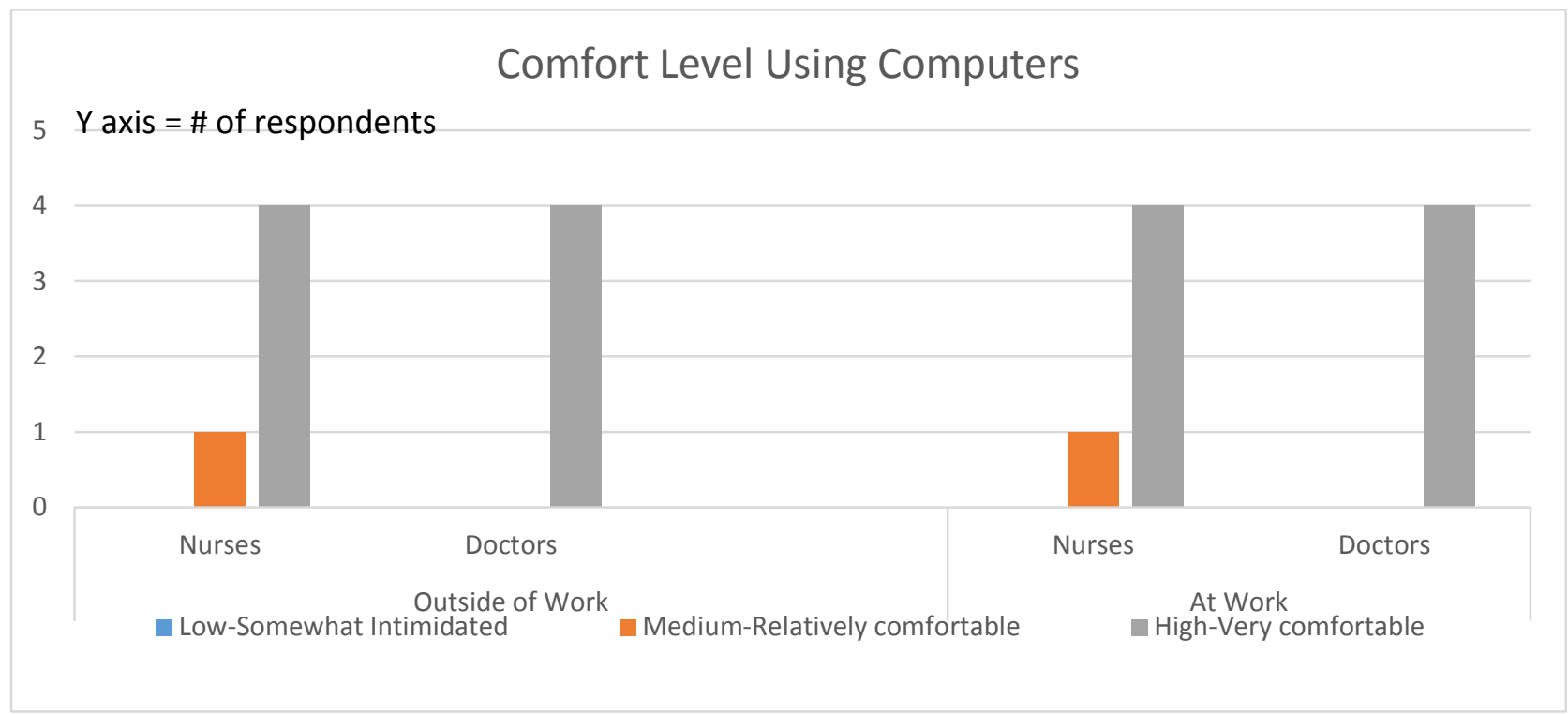

Table 2: Proficiency Level Using Computers

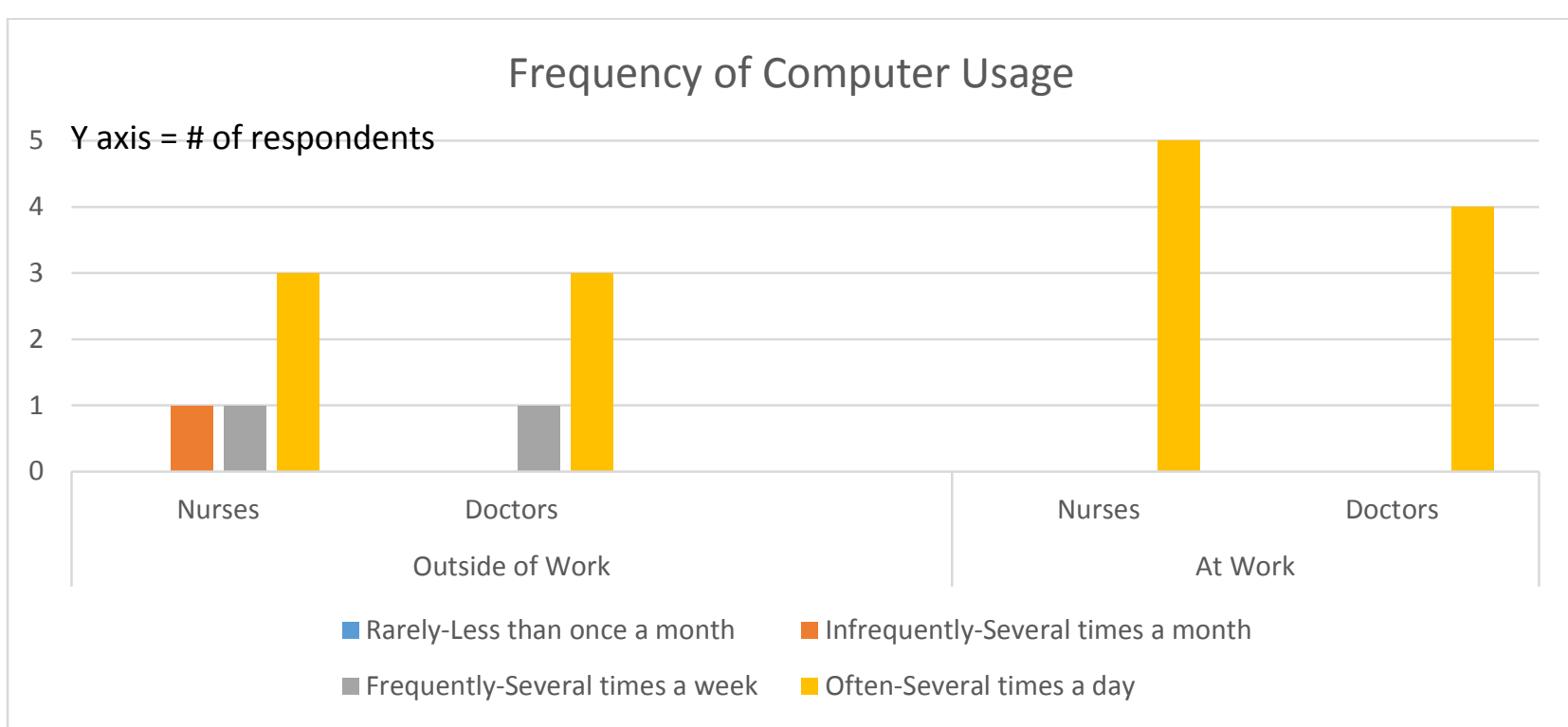

Table 3: Frequency of Computer Usage

This data indicates that participants are a relatively computer literate group. 
Types of devices used to interact with the EHRs:

An understanding of the devices used for and the mode of interaction with the EHRs may provide some understanding of the types of mistakes participants are likely to make when inputting data using functions such as the 'copy and paste'. Hence, the demographic questionnaire in our study asked participants what types of devices they used and what mode of interaction was used when engaging with their EHRs; and the participants' responses are represented in Tables 4 and 5, respectively.

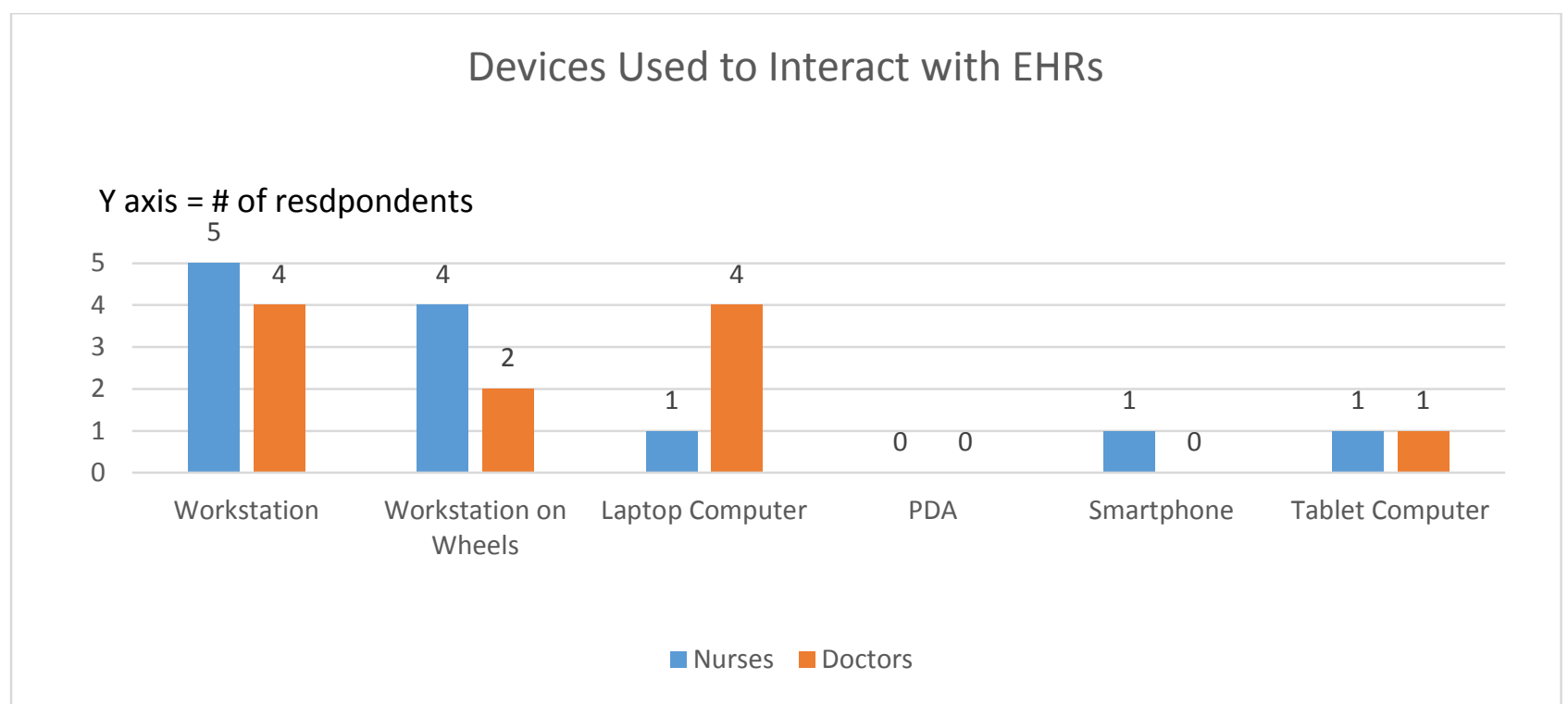

Table 4: Types of Devices Used to Interact with EHRs

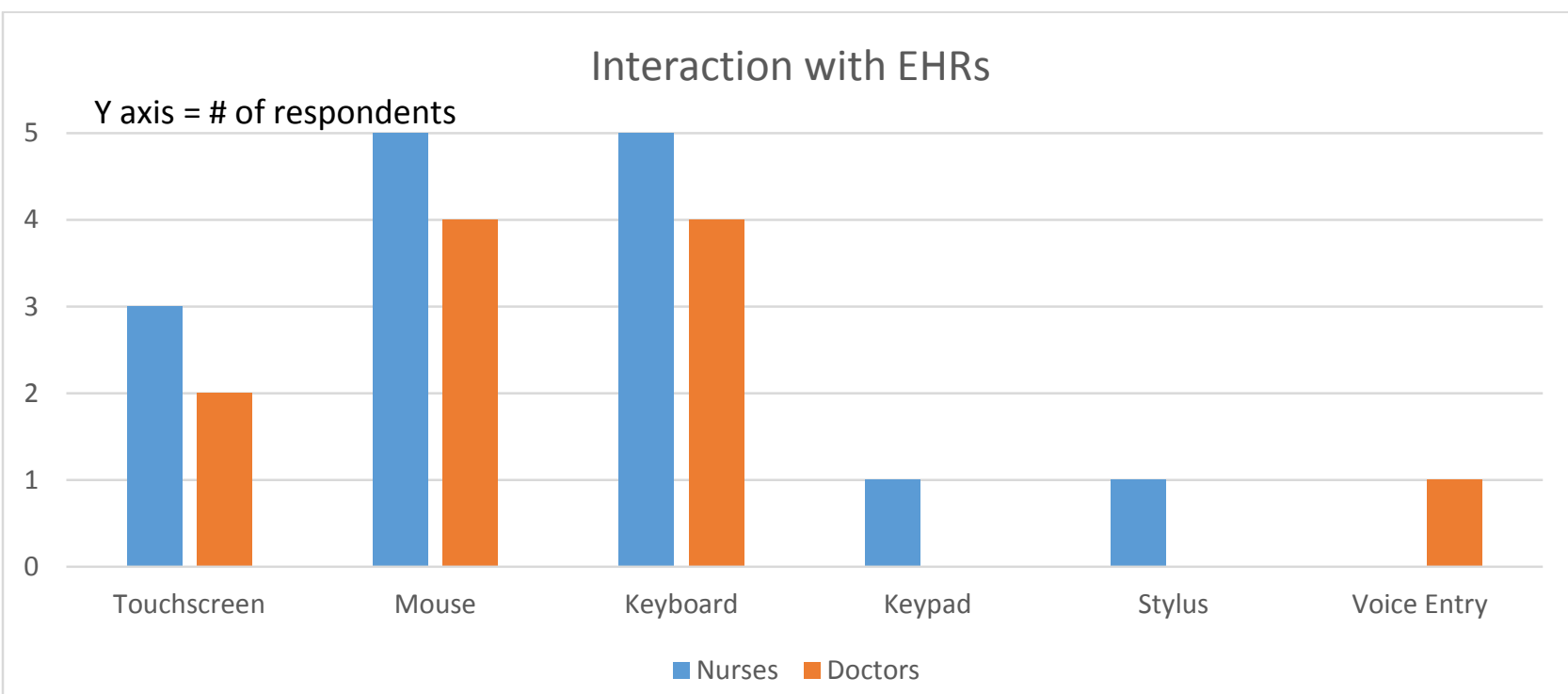

Table 5: How Participants Interact with their EHRs 
Not surprisingly, all of the nurses and physicians used a workstation when interacting with their EHRs, and four nurses and 2 physicians used a workstation on wheels. More number of physicians ( 4 of the study participants) than nurses (one study participant) use a laptop computer, and very few used a smartphone (one nurse) or tablet (one nurse and one physician). Again, perhaps not surprisingly, all nurses and physicians use a keyboard and a mouse when interacting with EHRs, with a touchscreen being the next most widely used ( 3 nurses and 2 physicians). One nurse reported using keypad, one nurse reported using stylus, and one physician reported using voice entry.

\subsubsection{Data Analysis}

Qualitative analysis of the observation and interview data began with the construction of a priori code list that was informed by prior research conducted by NIST ${ }^{1}$, the ECRI Institute convened Partnership for Health IT Patient Safety with respect to the 'copy and paste' functionality ${ }^{3}$ and other pertinent literature related to information integrity, qualitative research issues and EHR patient safety goals $6,7,8,8$. Also, the research questions and structural codes related to them helped the construction of the priori code.

One qualitative research expert performed the coding with the help of clinical experts (nursing and physician expertise). All codes were operationalized and delimited. After reading the full data set, a first round of coding was conducted utilizing the constructed a priori code list. This process also resulted in the identification of emergent codes from the data. The code list was revised appropriately. This open coding process applied labels to chunks of data in a reduction process that allowed for identification of like pieces of data. Multiple readings of the full data set occurred during this open coding phase, until saturation was reached, at which point no new properties or dimensions emerged. In the second round of coding, the goal was to identify relationships and connections among the open codes. This provided a way to develop conceptual categories that linked the codes and associated data. As data analysis proceeded, written coding memos provided another tool for thinking about and processing the data. Lastly, selective coding identified core variables and emergent themes that occurred across the data set.

\footnotetext{
${ }^{1}$ Lowry, S.Z., et. al. (2015). Technical Evaluation, Testing, and Validation of the Usability of Electronic Health Records: Empirically Based Use Cases for Validating Safety-Enhanced Usability and Guidelines for Standardization. NISTIR 78041.

${ }^{3}$ (2016). Health IT Safe Practices: Toolkit for the Safe Use of 'copy and paste'. Partnership for Health IT Patient Safety. https://www.ecri.org/Resources/HIT/CP Toolkit/Toolkit_CopyPaste_final.pdf

${ }^{6}$ Bowman, S. (2013). Impact of electronic health record systems on information integrity: Quality and safety implications. Perspectives in Health Information Management, 1-19.

${ }^{7}$ Noble, H., et. al. (2015). Issues of validity and reliability in qualitative research. Evidence Based Nursing, 18, 2, pp. 3435 .

${ }^{8}$ Sittig, D., et. al. (2012). Electronic health records and national patient-safety goals. New England Journal of Medicine, 367, 19, pp. 1854-1860.

8 (2011). AHIMA Copy Functionality Toolkit - A Practical Guide: Information Management and Governance of Copy Functions in Electronic Health Records.
} 


\subsubsection{Examples of Codes, Categories, and Themes}

In qualitative research, a code "symbolically assigns a summative, salient, essence-capturing and/or evocative attribute for a portion of language-based or visual data ${ }^{7}$." Coding is a cyclical act that serves as an intermediary between data collection and data analysis. It is argued that qualitative codes capture essential elements of the data and when those elements form clusters they can "facilitate the development of categories and thus analysis of their connections ${ }^{9}$." Codes are rooted in the data, but as coding and analysis continue, codes and categories become more refined and often, more conceptual and abstract. A further shift is evidenced "when the major categories are compared with each other and consolidated in various ways, you begin to transcend the "reality" of your data and progress toward the thematic, conceptual and theoretical." The examples below in Table 6 show how codes were applied to particular pieces of interview data (note that one chunk of data might have multiple codes applied to it). Ongoing analysis then examined the relationships among the codes to identify broader categories and later to identify emergent themes that ran across data sets.

This report focuses specifically on participants' perceptions of and experiences with the 'copy and paste' function in the EHRs. While data was coded for other ideas and issues related to EHRs, only data pertinent to the 'copy and paste' function was analyzed beyond first coding cycle.

${ }^{7}$ Saldana, J. (2009). The coding manual for qualitative researchers. SAGE. 


\begin{tabular}{|c|c|}
\hline DATA & CODE(S) \\
\hline $\begin{array}{l}\text { "I think it ['copy and paste' function] definitely saves time and improves efficiency. } \\
\text { And again, I think that I would say yes, I think physicians should be able to do it. Of } \\
\text { course, this is, at least in my mind, under the ethical role and almost self-accountability } \\
\text { of making sure that you're not actually just continuing to copy the same thing that was } \\
\text { done previously, as far as the daily progress." (P104) }\end{array}$ & $\begin{array}{l}\text { - Reason for Use } \\
\text { - Accountability }\end{array}$ \\
\hline $\begin{array}{l}\text { "Yeah because when you're doing a 'copy and paste', one of the problems is it's easier } \\
\text { to truncate something. So, I could have left off a word or got-- in this case it was so long } \\
\text { by the time you get to the bottom, the top is off the screen and you don't know if you } \\
\text { might have missed the first sentence or whatever. So, there are potential errors there. } \\
\text { And then when you copy it into the other place you don't reread it and later notice that } \\
\text { you've dropped something out..." (P101) }\end{array}$ & $\begin{array}{l}\text { - Possible Error: } \\
\text { Truncation } \\
\text { - Patient Safety }\end{array}$ \\
\hline $\begin{array}{l}\text { "In the transfer, yes, so it can be a use error on the person who's actually trying to input } \\
\text { the data because you have a lot of noise behind you when you're getting busy. If I'm } \\
\text { trying to copy information and put in, I could easily get something wrong. Just like say, } \\
\text { for example, the height. It went up two inches, but if I'm thinking in my head } \\
\text { everything's the same I could copy the same. Are you really going to add two inches to } \\
\text { the original?" (P202) }\end{array}$ & $\begin{array}{l}\text { - Possible error: Similar } \\
\text { but, not the same } \\
\text { - Patient Safety }\end{array}$ \\
\hline $\begin{array}{l}\text { "You don't, that's the thing about it. You don't know that you've copied and pasted, } \\
\text { because the way that the note is set up, it's just going to look like you just typed it out, } \\
\text { and it's going to let you edit it under the note section. Where you 'copy and paste' it, it's } \\
\text { not going to have any copyrights, or it's not going to have anybody that's signed it, } \\
\text { because your signature is the 'Okay' button. So it's not going to say, "This was written } \\
\text { by such and such." It will have that on a main source, but when you copy it, you're just } \\
\text { copying the portion that you want to potentially select." (P201) }\end{array}$ & $\begin{array}{l}\text { - Attribution } \\
\text { - Accountability }\end{array}$ \\
\hline $\begin{array}{l}\text { "Well, you're putting all this information in that's probably not accurate anymore. Like } \\
\text { what I did, I copied and pasted and I thought that I hadn't copied it all, so I went back. } \\
\text { Well, what if I didn't go back and I had copied only half of it? That's easy to do. I } \\
\text { always am uncomfortable about doing it, but that doesn't keep me from doing it } \\
\text { [chuckles] because then you're stuck typing out all this stuff that you already did." } \\
\text { (P205) }\end{array}$ & $\begin{array}{l}\text { - Possible error: Truncation } \\
\text { - Patient safety } \\
\text { - Reason for use }\end{array}$ \\
\hline $\begin{array}{l}\text { "I think the biggest thing about 'copy and paste' is bringing old information, that isn't } \\
\text { current, you don't recognize it and then don't edit it, then somebody else can enhance on } \\
\text { that. They don't know that-- then if you do a huge volume of 'copy and paste', until it's } \\
\text { kind of a secondary. You have somebody else reading it. If you're following a wrong } \\
\text { and a vital sign and you copied that in, it's highly unlikely the vital sign is the same. It's } \\
\text { probably one or two different or something like that. It may or may not be the-0- then I } \\
\text { guess the other thing is somebody bringing forward lab values. So you 'copy and paste' } \\
\text { bring forward lab value or weight that's now three months old and it looks like it could } \\
\text { be today's. Again and somebody acts on that data." (P101) }\end{array}$ & $\begin{array}{l}\text { - Possible error: old data } \\
\text { - Patient safety } \\
\text { - Accountability }\end{array}$ \\
\hline $\begin{array}{l}\text { "... while I was kind of trying to get through the task - put in the information and update } \\
\text { it based on Brody (synthetic computer-generated patient name) instead of Tatiana } \\
\text { (synthetic computer-generated patient name) - there's a high likelihood that some of the } \\
\text { information that was previously on Tatiana's note would then end up being on Brody's } \\
\text { note, without me knowing." (104) }\end{array}$ & $\begin{array}{l}\text { - Possible error: Wrong } \\
\text { patient } \\
\text { - Patient Safety }\end{array}$ \\
\hline $\begin{array}{l}\text { "Part of it if you just have the attribution, the thing I talk about is in Outlook, if you } \\
\text { copy an email into a new email it usually changes color. It may not have the meta-data } \\
\text { along with it, but at least it changes the color so that you can see, ....Hey this was } \\
\text { probably... Well if you're a student looking at that, you see that that was brought over. } \\
\text { But like I said, ideally, it would say, copied from a month ago." (P101) }\end{array}$ & $\begin{array}{l}\text { - Possible improvement: } \\
\text { Attribution; highlight }\end{array}$ \\
\hline
\end{tabular}

Table 6: Examples of Data and Codes 
As coding continued, several larger categories developed, including the importance of time and reliability in the use of the 'copy and paste' function. As selective coding progressed, subthemes of Finding Information, Moving Information, Understanding Information and Using Information emerged. These themes were all tied to an overarching theme of Integrity of Information. A discussion of these categories and themes is presented below in the Results section. 


\section{Study Results}

This section presents an analysis of the data related to the 'copy and paste' function in EHRs from the nine participants in this study. Initially, structural codes related to the research questions focused on reasons for using the 'copy and paste' function, problems with using the 'copy and paste' function, possible errors related to the use of the 'copy and paste' function, and the potential safety issues related to the use of the 'copy and paste' function. These structural codes, while informative, only begin to tell the story of the data and are surface level in their representation of data and their use as reduction tools. The second cycle of coding, known as the axial coding, provided a more nuanced view of the data and broader categories, highlighting the importance of "Identification" and "Reliability" in the use of the 'copy and paste' function, which cut across the structural codes identified in the first cycle of coding. The overarching theme that tied all data together was the "Integrity of Information," which related to finding information, copying information, understanding information and reusing information.

Participants' perceptions of and experiences with the 'copy and paste' function lead them to believe there is a greater likelihood of error and possible safety risks for patients when it is used. In the following sections, these categories and subthemes, and their relationship to error and safety are explored. The participants and data referenced here are used as exemplars for highlighting the themes and major ideas. They are by no means unique statements, but are representative of the study findings as a whole.

\subsection{Integrity of Information}

One common thing among the reported perceptions and experiences of participants pertaining to the use of the 'copy and paste' function was the "Integrity of Information." Even when speaking of the potential benefits for using the 'copy and paste' function, concern about integrity surfaced. For example, many of the nurses spoke about how much more reliable information is when it is copied and pasted than when it is typed in from memory. Participant P202 notes this quite succinctly.

"It's helpful, because I feel if you were able to 'copy and paste', even

most of the items or what not, at least I know what's coming over is more accurate than me just typing it back out."

Of the nurses, only P201 preferred to always type data in by hand and often, from memory rather than utilizing the 'copy and paste' function.

"What some people do is, if an incident occurred on one day and they have a similar incident, they'll copy it even though it may only have some similarities to where you are pasting, you still want to do a flat out new report, no matter if its similar or not. And, a lot of people get caught up that way with copying and pasting something that's similar, instead of just doing a whole new report. So, it can get tricky that way. Someone had done that before, and it was - they didn't really read through it all. It was a little piece of information that totally had nothing to do with this new incident. So it's best to just have a fresh start for everything that you do. So that's the downfall to copying and pasting, because people take advantage of it."

While several participants recognized that using the 'copy and paste' function might save them time or be more efficient, all participants articulated a recognition of the ways the integrity of information in the patient's chart may be compromised by the use of the 'copy and paste' function. This loss of integrity using the "copy and paste' function is related to four areas: "Finding Information," "Copying 
Information," "Understanding Information" and "Reusing Information" (see Figure 1 below). All four areas provide opportunity for the introduction of error/s which potentially can lead to patient safety issues. Each of them, along with the subcategories of "Identification" and "Reliability," are discussed in the following sections.

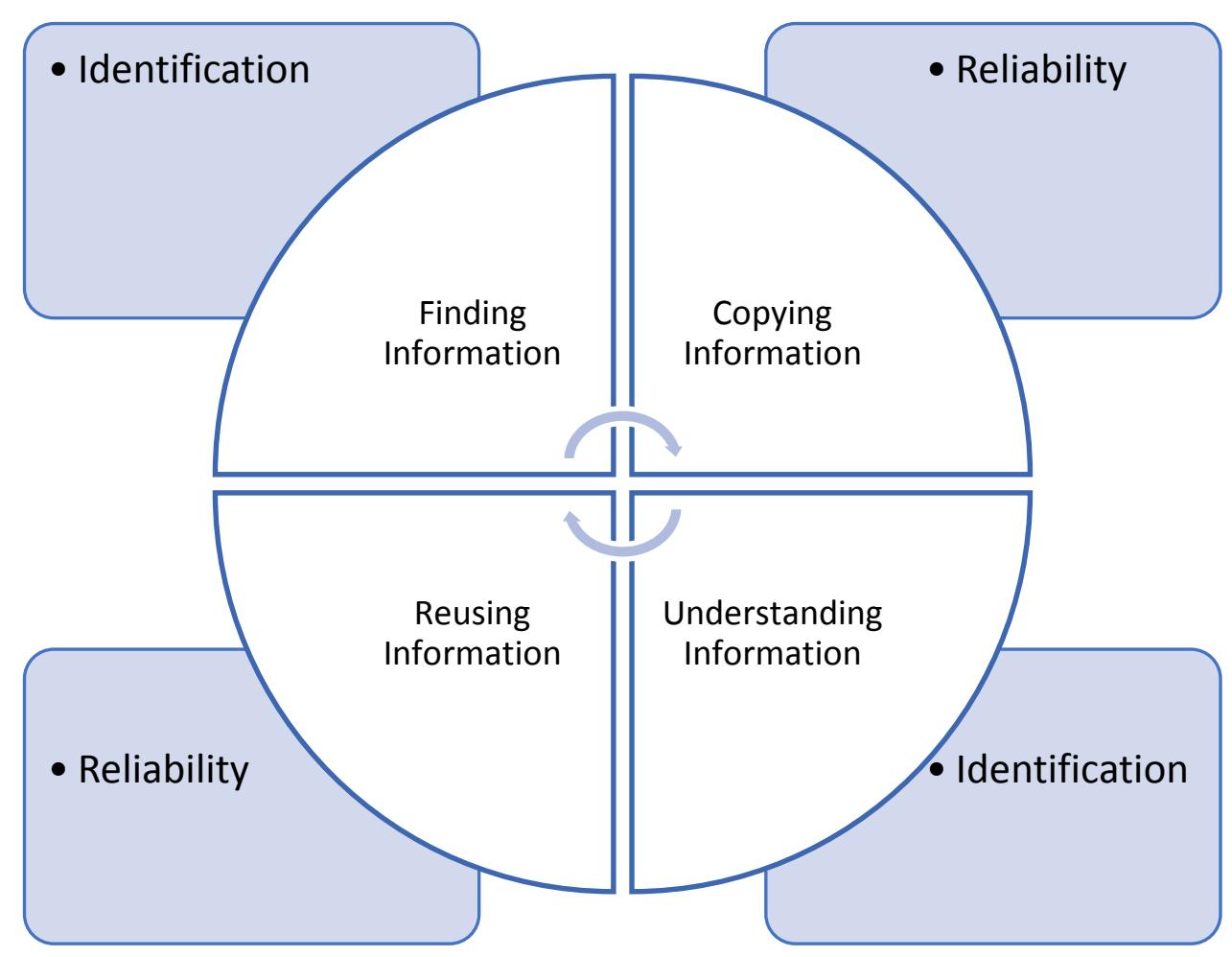

Figure 1: Model of Issues Resulting from the 'copy and paste' Function

\subsection{Identification and Reliability}

Both of these codes, "Identification" and "Reliability," had positive and negative associations. Time was one of the reasons given by many participants for using the 'copy and paste' function, like, as noted by P201:

'It's just for the times' sake, if it's like very, very busy or something like that." 
Several participants, however, stated that when notes are copied and pasted "note bloat" (P101) often occurs and that when this happens, much more time is required to read and process the information in a patient's chart, which can waste the practitioner's time, as evidenced in the following statement.

“...you could 'copy and paste' it, but you want to enter not as much information to the doctor to have to read over. You want to summarize it up so he [the doctor] can look at it, know exactly what's going on, and keep it moving. You don't really want to overwhelm him with a big paragraph of stuff that he has to go through. It takes too much time. Everything in healthcare is time. Time is very important." (P201)

In the quote above, the same participant who recognized the benefit of using the 'copy and paste' function to help save time in documentation when she is busy, also recognized how her copying and pasting large amounts of the information might "overwhelm" a physician and end up taking "too much time." She acknowledges that a clinician's effectiveness might be diminished as a result of having so much information in the record.

Physicians and nurses alike demonstrated in their responses that the person who copied and pasted saved time at the expense of the person who had to spend extra time reading the copied material. P101 noted how the use of the 'copy and paste' function often results in "hyperdocumentation."

"They've absolutely not contributed to helping the physician-patient interaction and in most cases you've got what amounts to and what I would call hyperdocumentation. The fact that you can click one button and it generates all page of either negatives or something to the point that they've become too cumbersome to use when you look back at what their history was over the last couple of months or couple of years. It's just too much information to be useful." (P101)

All of the physicians and four out of five nurses mentioned how the use of 'copy and paste' function often results in "note bloat" that takes an inordinate amount of time to read and process, resulting in less efficiency since it takes additional time to identify relevant and accurate information. While P101, a physician with many years of experience in his field and using EHRs, recognized the peril of "hyperdocumentation" - that there is "just too much information to be useful" - during the scenario and usability task portion of the interview he himself used the 'copy and paste' function to reuse information into a note and in the post-task questions he stated

"...that's one of the advantages of electronic health records, once it's in now you can use it many times."

Participants also spoke of how the reliability of information is often diminished in the situation when a chart is filled with "too much information." One doctor, in speaking about using the "copy and paste' function noted how some significant information "would just be buried in the text" (P104), making it difficult to find and thus more likely that a practitioner would miss it, as explained in the statements below from P103 and P104.

"Let's say a patient was on a certain medication or has a certain exam

finding on day one and you copy forward that note to day two. What you miss - and that patient no longer has an exam finding or is on that medication - but you miss that part of the note where you're stating that that patient is on that medicine or has that exam finding you just alter the exam or the assessment and plan. Well, then it appears by the medical 
record with your signature on it that that patient was on the medication for a lot longer than the patient or have that exam finding for." (P103)

"I think data overall then does not get adequately reviewed on a daily basis, and so things sometimes get left in a chart, expires text or certain things if a physician is not careful about changing that on a daily basis. Eventually, that could lead to a safety error if say, a different physician, a different provider then tries to go through what documentation is available and base their decisions on what is there." (P104)

In these situations, inaccurate information or information that is no longer relevant remains in records, keeps getting copied, and possibly obscures other relevant information. This interference can result in errors as evidenced by P102's account of a situation a friend of his faced.

"Sure. Here is a fresh one. A friend of mine... saw a patient and somewhere on page three of their record there was a reference to a suspected cancer and a pathology result that nobody saw. And so he learned about it from an attorney on behalf of the person."

All of the physicians and three of the five nurses made reference to similar stories or ideas - that the use of the 'copy and paste' function often results in the obfuscation of important information that could lead to error and could compromise patient safety.

However, one of the physicians also spoke at length about how the "judicious and accountable use" of the 'copy and paste' function could provide more reliable information.

"So I think that there's, generally speaking, in my experience, in the world of medical informatics, when people make any reference to 'copy and paste', they say it because people copy entire notes from some past visit. They clearly don't read the whole thing. They paste the work they've scanned. Well, they may have read it, but at the time they do paste and sign the chart, they haven't re-read it and they certainly haven't edited it. They haven't taken out the things that they didn't directly observe themselves. They generally don't type in, 'This is something that I 'copy and paste' from the last notes. I didn't actually examine the patient, but that's what they found last month,' and so-- they don't go there. And then doctor's experience, when they see something that's pasted and it's clearly not something that the person did who pasted it, and they're like, 'this is horrible. 'Copy and paste' should be just disabled from all electronic health records, period. It's just too likely for people to do unethical things.' That's generally the context. So that's counter to judicious and accountable use of 'copy and paste' from the standpoint of you're more likely to get the right information in accurately if you use it responsibly." (P102)

He recognized that problems occur when the 'copy and paste' function is not used judiciously, for example, when people "copy entire notes from some past visit" or "don't read the whole thing" or they "paste work they've scanned." However, he still believes that "you're more likely to get the right information in accurately" by using the 'copy and paste' function. In many ways, this highlights what several participants noted is the importance of accountability in the process of using the "copy and paste' function, something that will be discussed later in this report. 


\subsubsection{Finding Information}

Participants believed that finding information was connected to identification - of both the patient and of relevant information about the patient. As noted above, some participants believe the 'copy and paste' function provided a means to reuse information quickly, efficiently and reliably. On the other hand, they often articulated the ways in which doing so created situations that had the potential for error. For example, several participants noted the importance of making sure information that was being copied was pasted into the correct chart. P201 and P202 describe below what can happen when using the CPF in a hectic environment when multiple charts are open.

"...but the thing that can get confusing is because some people have more than one patient's chart up at one time, and if you 'copy and paste' something from another person's chart, and you are not looking at the note that you add, it can get confusing and put this information with another patient." (P201)

"If you have six patients-- I've had 20-some patients at the rehab center, so you walk through those patients. It's so easy to click on the wrong person or someone's sharing a room. Or you're walking back and forth because you decided, oh, somebody needed pain medicine. Let me go to them. Yeah, it's just chaos. Definitely, charting on the wrong patient is huge and if they realize, then they have to go back and say, 'This is the wrong patient data. This does not belong to patient such-and-such.'..." (P202)

In these situations, it is imperative that practitioners find and are working in the correct chart when utilizing the 'copy and paste' function. Similar issues were reported in NISTIR $7804^{10}$, which resulted in recommendations to reduce the risk of entering incorrect information in the wrong chart.

In addition, as discussed in the section above on "Reliability," participants recognized the ways in which the use of the "copy and paste' function often resulted in "hyperdocumentation" and "note bloat," making it difficult to find accurate, relevant and up-to-date information about a patient. Again, the NISTIR 7804 document provides guidance here, noting that EHRs should "support efficient and easy identification of inaccurate, outdated or inappropriate items." This would alleviate the situation discussed by P204 below.

"... because the doctors' notes that they do, where it would be helpful to know because they just copied and pasted this as a general overview of what the kid has had going on, but it's not accurate as to this past 24 hours. That does get really sticky sometimes because they'll say they're on-- It makes you question-- You'll see this plan for today is, "Stop," or "Stop this medication," "Start this," whatever. So in my head, I'm making out my to-do list like, "Okay, I should stop this medication today," but if you don't clarify and they might say, "Oh, we already stopped that yesterday or no, we're stopping it tomorrow." (P204)

All of the participants articulated something similar that highlighted the ways in which the "copy and paste' function created a "sticky" situation where practitioners have difficulty in finding and identifying relevant information necessary for providing quality care to patients.

${ }^{10}$ Lowry, S.Z., et. al. (2012). Technical Evaluation, Testing, and Validation of the Usability of Electronic Health Records. NISTIR 7804 . 


\subsubsection{Copying Information}

While participants recognized the positives in using the 'copy and paste' function, they also recognized the ways in which reusing information in this way might result in less reliable information. One reason for this concern is the ease with which a user might truncate information, not capturing everything they meant to copy.

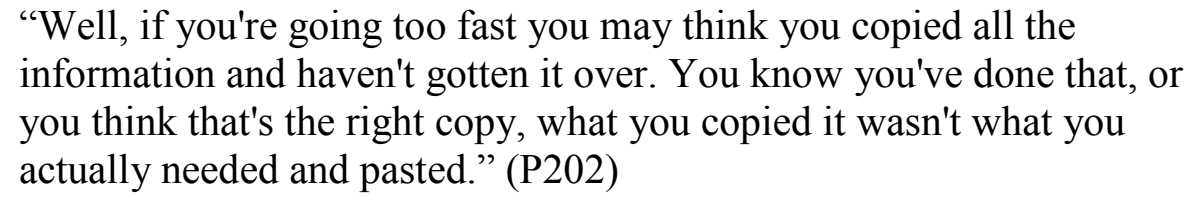

In order to ensure the reliability and integrity of the information they 'copy and paste' into the patient record, users need a way to know when truncation of that information has occurred. They and their clinical colleagues depend on complete and accurate documentation in the patient record to do their work and provide safe, quality care for patients.

In addition to incorrectly copying information while using the 'copy and paste' function, many participants noted that when whole sections are copied and pasted, there is a need to go in and edit some of the information, since it is rare that everything documented in the prior note about the patient would stay the same for the current note- - for example with a medication list or vital signsinformation is often similar but not the same.

"Just if you're copying and pasting one information into another one then you could easily forget to change one number in a blood pressure or something." (P203)

"I think the biggest thing about 'copy and paste' is bringing old information, that isn't current, you don't recognize it and then don't edit it, then somebody else can enhance on that. They don't know that-- then if you do a huge volume of 'copy and paste', until it's kind of a secondary. You have somebody else reading it. If you're following a wrong vital sign and you copied that in, it's highly unlikely the vital sign is the same. It's probably one or two different or something like that. It may or may not be the same. Then, I guess the other thing is somebody bringing forward lab values." (P101)

These two statements are representative of participant comments related to the perils of moving information through the use of the 'copy and paste' function in an EHR. The 'copy and paste' function enables propagation of entire sets of information when parts of the information may or may not be the same for the current encounter. Without a means to prompt users to edit information that has been copied and pasted in its entirety, there is a likelihood that incorrect and irrelevant data will make its way into a patient's chart.

\subsubsection{Understanding Information}

Participants recognized that when notes and other patient data are copied and pasted, this information is not always read thoroughly due to the large volume of data created in the patient record. A practitioner's ability to understand someone else's terminology (including abbreviations and shortened terminology) often make it difficult to interpret previous notes and data. For example, P101 noted several times that it is important to keep in mind that "you are documenting for somebody else" and 
that what makes sense to you may not make sense to someone else. Later in the interview, he expanded on this idea, noting that:

"people free text, they come up with all sorts of innovative abbreviations and it tends to be their own. From my experience, I constantly come across abbreviations that I've never heard of. I've been practicing for 30 years and have no idea what it means. And so we've lost that information..." (P101)

Another participant remarked that users often resort to their own terminology (shortened terminology or abbreviations) in their documentation, thinking they themselves will be the only ones who will access and read the information. Then, when some other authorized person does access the patient record, the information that was documented is difficult for them to interpret.

\subsubsection{Reusing Information}

Several participants saw the use of the "copy and paste' function as "cheating" and as "taking the easy way out." Instead of taking the time and care to enter information themselves, users tend to just "copy and paste' information, at times, the information that has been documented by someone else.

"It can be a problem if you're trying to do things the wrong way or the easy way out. Some people will 'copy and paste' the information onto another screen and act as if they were the one that did the report." (P201)

"... we can just copy something from the day prior, or copy something from a previous interaction or chart. And then just put it in and claim it as our own from the day that we're actually interacting or documenting something." (P104)

These participants, felt practice of using the 'copy and paste' function resulted in information that is not reliable since the true source of the information is unknown. In two different sections of her interview, P201 discussed how if she does use the CPF she wants to make sure the information she charts in the patient's record is accurate because her name is on the submission, and she is ultimately responsible for it.

"So it can get very tricky, because you don't want to 'copy and paste' something that's not accurate, and then now your credentials is on it, and you're the one that's submitting it to the doctor. So you always want to double check and look through the patient chart and go over which you submitted if you're getting it from someone else." (P201)

"Exactly, because you will prefer to write down your own versus copying and pasting what someone else is putting. Because you know if something did go wrong, that you're the one who should be at fault. But if I 'copy and paste' it and they're wrong, I'm at fault still. But I'd rather be at fault for my own mistake than someone else's." (P201)

Using information from others, that has been copied and pasted, poses a threat to the copier's work, reputation and liability, especially if an error occurs and patient safety is affected. 


\section{Empirical Evidence to the Partnership Recommendations on 'copy and paste' Function Use}

Based on a comprehensive investigation of the literature and data related to the use of the 'copy and paste' function, the ECRI Institute convened Partnership for Health IT Patient Safety created four recommendations surrounding the use of the 'copy and paste' function in EHRs.

Recommendation A: Provide a mechanism to make 'copy and paste' material easily identifiable.

Recommendation B: Ensure that the provenance of 'copy and paste' material is readily available.

Recommendation $\mathrm{C}$ : Ensure adequate staff training and education regarding the appropriate and safe use of 'copy and paste'.

Recommendation D: Ensure that 'copy and paste' practices are regularly monitored, measured, and assessed.

Data from this NIST study overwhelmingly supports Recommendation A and Recommendation B, as discussed below. There is no direct data to report Recommendation C and Recommendation D, although there are some general indicators pointing toward ways in which the participants tangentially provided support for Recommendation $\mathrm{C}$ related to training.

\subsection{Recommendation A: Provide a Mechanism to Make 'copy and paste' Material Easily Identifiable}

Many participants provided feedback confirming the recommendation, giving examples of solutions similar to those provided by the ECRI Institute convened multi-stakeholder Partnership for Health IT Patient Safety. For example, to support Recommendation A, copied material could be:

- Seen in a split screen;

- Seen by hovering the cursor (which would help with screen clutter);

- Presented in a different font (for example, italicized, a different color, etc.);

- Presented in some way that is different than content entered by user.

Participants often said they thought that any information reused with the 'copy and paste' function should be visibly marked as having been copied and pasted.

"Yeah...some type of visual. So see if I copied and pasted that

information, even onto a note. When I put it in there, maybe it's yellow, because this was copied." (P202)

"I really think the highlighting thing. I think maybe you should be able to highlight and 'copy and paste' within one person's chart..." (P203)

"...the thing I talk about is in Outlook, if you copy an email into a new email it usually changes color. it may not have the meta-data along with it, but at least it changes the color so that you can see, 'Hey this was probably copied' Well if you're a student looking at that, you see that that was brought over. I think either change of color or highlight it or italicized or whatever, underlined..." (P101) 
"I think it would be tough for me to put it in and not be able to highlight it well. So that if someone else were to ever pull up this note again, they would actually realize and identify that." (P103)

Here participants offered several suggestions for how the copied data could be made more visible and visual and thus, more easily identified as having been copied and pasted. Across the data set, these ideas for possible improvement surfaced, all supporting Recommendation A that there needs to be a way to easily identify information reused with the 'copy and paste' function.

\subsection{Recommendation B: Ensure That the Provenance of 'copy and paste' Material Is Readily Available}

The participants, felt the practice of using the 'copy and paste' function resulted in information that is not reliable since the true source of information is unknown. In two different sections of her interview, P201 discussed how if she does use the 'copy and paste' function, she wants to make sure the information she charts in the patient's record is accurate because her name is on the submission, and she is ultimately responsible for it.

"So it can get very tricky, because you don't want to 'copy and paste' something that's not accurate, and then now your credentials is on it, and you're the one that's submitting it to the doctor. So you always want to double check and look through the patient's chart and go over which you submitted if you're getting it from someone else." (P201)

"Exactly, because you will prefer to write down your own versus copying and pasting what someone else is putting. Because you know if something did go wrong, that you're the one who should be at fault. But if I 'copy and paste' it and they're wrong, I'm at fault still. But I'd rather be at fault for my own mistake than someone else's." (P201)

Participants also provided overwhelming support for this recommendation, talking about the need for "metadata" and information about where the data came from that is reused by the 'copy and paste' function. P101 and P102 provide the clearest articulation of this

"But like I said, ideally, it would say, "Copied from a month ago." And so you could have something like track changes that showed what you edited this time. And it might-- that sort of functionality might force people to not do it because it shows exactly what you've done, and then it ends up looking ugly with all these different colors and things like that and having the metadata copied from and sometimes people are copying from somebody else's note." (P101)

"If I use that example, again, of 'copy and paste' and the expectations that I have, we can expect that there would be multiple ways to do it. Should there also be an expectation in terms of safety that material that's copied and pasted, either should be highlighted, or should be attributed, or meta-data should transfer with it.... I would expect - this might sound - I would want to be able to, from a policy standpoint. Say,"Of course, we're going to have traceability on how those things got into that note." (P102) 
Having "traceability," as P102 says, is imperative in the use of the 'copy and paste' function if we care about the integrity of the information and the safety of patients. The ECRI Institute convened Partnership for Health IT Patient Safety provides several examples of possible solutions, many of which participants discussed. For example:

- Providing a log of when notes are changed, who changed them, and how they were changed;

- Creating the possibility for information about copied material to appear in a separate window;

- Identifying the provenance of copied information when the cursor hovers;

- Having metadata available with copied information.

Data from this study overwhelmingly support these examples, and both doctors and nurses believe the source of the copied information should be readily available.

\subsection{Recommendation $C$ : Ensure adequate staff training and education regarding the appropriate and safe use of 'copy and paste'}

Our study confirmed the importance of training for the use of the 'copy and paste' functionality. Most of the study participants reported the fact that training raises awareness of the error prone actions while using the 'copy and paste' function. Some of the participants in our study were not aware if the EHRs at their workplace made available the 'copy and paste' functionality. They quoted that training and education regarding the 'copy and paste' function would increase their efficiency as well as help them use the functionality in a safe way. They thought that proper training helps reduce errors that can occur as a consequence of improper use of the 'copy and paste' functionality.

In our testing protocol, the participants were first asked to watch an instructional video about the EHR they were using for the test. Then, test participants were given instructional material to familiarize themselves with the EHR used in the study. On the side note, it was observed during the task performance that participants learned instructions better by watching the video than reading printed material. Also, those participants who were more attentive to the instructional part of testing session, performed more accurately and efficiently while accomplishing the tasks than the participants who mostly relied on their general knowledge of the EHRs. 


\section{Human Factors Assessments of the 'copy and paste' Function}

Our study revealed that integrity of information as the major concerning factor while using the 'copy and paste' function. 'Copy and paste' function increased the efficiency with regard to managing time, but can lead to errors when the information gets truncated, when the information is old, if the information came from another individual, if the information was erroneous to start with where the error gets propagated, etc. The legibility of information on the screen could be impaired by complex layout presentation which could trigger 'copy and paste' errors. For example, when the end user copies content from a chart (with the gridlines and alignment) in the EHR into a document, if the formatting is lost, there is a negative impact on the semantics of the text/information. Our research recognized the following key aspects related to the 'copy and paste' function based on the human factors assessment derived from empirical data obtained.

1. Amount of information: Due to the ease of extracting a large amount of information, the users could be presented with convoluted information and increased complexity of the user interface. Convoluted information is the result of too much text related to hyperdocumentation / note bloat. Hyperdocumentation / note bloat obscures relevant information and makes it more difficult for clinical providers to extract important information. Situation awareness is impaired with excess and/or irrelevant information during hyperdocumentation / note bloat. Users may miss important pieces of information.

2. Attribution of information: There was overall consensus among the participants regarding the notion of attribution. Clinicians need to know the source of the copied and pasted information, who copied and pasted it, what was added to/edited in the information, and the date and time the information was copied and pasted. Our research showed behavioral differences between doctors and nurses who use the 'copy and paste' function. Nurses are typically, under peer pressure and therefore, are not inclined to use the 'copy and paste' function in the EHR, while most of the time, the physicians not under any peer pressure associated with time constraints to document elaborate patient information, use the function of 'copy and paste' extensively.

3. Wrong information: The 'copy and paste' function can be efficient as well as safe, if the user is diligent in proofreading and editing. Most commonly though, the users forget to edit the information they have copied and pasted (interruptions being one of the major reasons) or are not provided with features that promote efficient editing. There were instances where the EHR did not allow the users to change the copied and pasted information - especially the times when medication was administered versus when it was charted. Also, lab results are only allowed to be copied in complete sets, so the pertinent information-e.g., the information that may be relevant for determining the diagnosis is obscured, and the lab information that can be copied generally cannot be edited.

Efficiency in the utilization of information is dependent on the following four critical aspects:

- Finding information

- Copying information

- Understanding information

- Reusing information

The documentation of healthcare information includes complex content and can be bulky, as in surgical procedures. Documentation of such enormous, but important information can become 
burdensome if the ability to reuse the information with the use of functionalities like 'copy and paste' and/or carry forward are not available in the EHR. This can decrease the efficiency of the clinical users who are constantly under time pressure and can lead to inadequate care delivery. This was demonstrated during our testing session where one of the physicians was persistent in his actions to 'copy and paste,' because his task was to reproduce a large set of information (e.g. in a discharge summary). He presumed that manually re-entering the information was both time-consuming and error-prone. In his case, the EHR employed in the study required workarounds to use the 'copy and paste' function in certain sections. Despite this fact, the physician was successful in copying and pasting to accomplish the particular task. The providers who are unable to 'copy and paste' in a reasonable manner become frustrated. Thus, the function of 'copy and paste' should be made available in the EHR wherever it is considered to be safe to do so. We hope the recommendations provided as part of this research serves this purpose. The hospitals should necessarily, be given the ability to turnoff the 'copy and paste' function in certain areas of the EHR.

\subsection{Human Factors Recommendations}

In this section, we provide general recommendations, followed by some specific recommendations for the user interface design to ensure safety-related usability of the 'copy and paste' function. The human factors recommendations provided below based on the empirical research, complement the ECRI Institute convened Partnership for Health IT Patient Safety's recommendations for the safe use of 'copy and paste' function.

\subsubsection{Human Factors Recommendations to support The Partnership Recommendation}

A: Provide a mechanism to make 'copy and paste' material easily identifiable

EHR systems must be designed to enhance the visibility of the information being selected for 'copy and paste' to prevent users from inadvertently copying only part of the information that was intended to be copied which could minimize the possibility of incomplete reuse of information that could lead to morbid/mortal errors.

Incomplete information documented, in particular, during copying and pasting, could lead to morbid/mortal errors. To minimize this possibility, the EHR system must make available provisions that can enhance the visibility of the information to help the user avoid inadvertently copying and pasting only part of the information that was intended. Many participants in the testing session mentioned that the information they wish to reuse, needs to be provided in a separate field within the same screen either by means of a split screen or a pop up window that helps their documentation process.

$>$ EHR systems should provide a concept for reconciling that the copied information was read consciously and edited by the clinical provider which would promote the attribution of the source of the information.

In our study, both observation and interview data strongly suggest the requirement for robust ways to edit the copied and pasted text. While editing, the user can forget where he/she stopped reading because of the widely prevalent interruptions in medical practice. One example could be, at the first instance of pasting the copied information, the text appears in a color other than the normal text. After reconciling (it could be a pop up over the highlight asking for reconciling 
the edited information) the text turns to the normal text color but has a "chain of custody" viewable by the clinical provider on demand.

Areas that should be locked from copying:

- The 'copy and paste' function must not be allowed when entering any information into a blood bank information system due to the extreme risk involved in blood transfusion.

- Demographic information: Demographic information should never be copied, but needs to be autopopulated in all the interfaces within a patient's chart.

- Copying of the demographic data from one chart to another should NOT be allowed. NIST research revealed a clear trend in the users' perception that each subsection of the patient chart needs to be autopopulated with the patient demographics. However, there were instances mentioned where manual entry of the demographic data becomes necessary during documentation outside of the EHR platform. Necessary provisions need to be provided in these instances to ensure correct entry of the demographic information.

- Dates should never be copied and pasted.

\subsubsection{Human Factors Recommendation to support The Partnership Recommendation B: Ensure that the provenance of 'copy and paste' material is readily available}

User interface must display the "chain of custody" of the information associated with the use of 'copy and paste'. However, this information should not be displayed by default, and only be shown on user demand to avoid the possibility of overwhelming clinical users and contribute to errors of commission (taking an incorrect action).

The 'copy and paste' function, as such, may result in too much text due to hyperdocumentation / note bloat that leads to convoluted information. To avoid overwhelming the clinical users any further, with excessively complex presentation in the interface, the "chain of custody" information associated with use of 'copy and paste' should not be displayed by default, but should only be shown on user demand. This is to avoid distracting the users with any additional data that might overwhelm them which could contribute to the errors of commission.

\subsubsection{Additional Human Factors Recommendations for critical areas identified in the study}

Vital Signs

The following properties should be displayed when documenting vital signs through charting done by the clinical user or by real time feed to the EHR system from digital devices:

- $\quad$ Time stamp when measured. How it was measured (nurse manually takes BP, arterial line captures BP at a single time)

- Time stamp when documented in the EHR.

- Time stamp when it is signed (locked).

- Time stamp when it is revised.

- Time stamp when retrieved. 
For the purpose of data traceability, whether the digital medical device feeds the data into the EHR or the user copies and pastes the data, the originator of the data elements should be identified along with an electronic date and time stamp at the time the data is entered in the sponsor's electronic system ${ }^{11,12}$.

\section{Allergies}

There should be clear "chain of custody" when copying and pasting information related to allergies.

Accurate recording of allergies is critical in patient care (within a single setting or across settings within an institution). This is due to the morbidity and mortality associated with potential errors coming from erroneous or inappropriate copying and pasting of the allergy information. For example, during the documentation of the transfer of care from one department to another, there can be risk of compromise in the accuracy of the information which could result in anaphylactic reactions of the patient. At all instances where 'copy and paste' function is used for the reuse of information during documentation of allergies, traceability with the source of the information must be included.

\section{Surgical notes}

The design of the EHR must ensure that all of the information pertaining to surgical notes is provided with the 'copy and paste' functionality which helps the reuse of information by the clinical staff in the "exact" and precise context of the surgical notes. Variability may exist due to patient-specific findings and/or specific situations that might arise unexpectedly during the procedure which must be accommodated by EHRs by means of provisions for efficient editing.

Surgical procedures are supposedly, performed in a typical fashion and the surgical notes are physician-dependent. Inevitably there will be situations where deviations from the norm are necessary during a given surgical encounter. Variability may exist due to patient-specific findings and/or specific situations that might arise unexpectedly during the procedure. To facilitate the complete and efficient entry of surgical notes, it is appropriate for the surgical and associated clinical staff to reuse information to document surgical procedures they perform frequently. The design of the EHR must ensure that all of the information pertaining to surgical notes is provided with the 'copy and paste' functionality which helps the reuse of information by the clinical staff in the "exact" and precise context of the surgical notes. The EHR also must ensure that the chain of custody or the text identifier which includes the original author, the date, and the source of information is available for display to the user, on demand. Since the surgical notes may vary depending on particular patient findings and/or unexpected situations arising during the procedure, strict editing requirements must be emphasized and/or reinforcement of strict editing of the copied and pasted surgical notes should be applied. The EHR must be designed to allow the entire text of the surgical note to be editable.

11 (2016). Use of Electronic Health Record Data in Clinical Investigations. U.S. Department of Health and Human Services Food and Drug Administration Draft Guidance for Industry. http://www.fda.gov/ucm/groups/fdagov-public/@fdagovdrugs-gen/documents/document/ucm501068.pdf

12 (2013). Electronic Source Data in Clinical Investigations. U.S. Department of Health and Human Services Food and Drug Administration Guidance for Industry.

http://www.fda.gov/downloads/drugs/guidancecomplianceregulatoryinformation/guidances/ucm328691.pdf 


\section{Medication Entry}

When medication entry is done in free text form, the 'copy and paste' function could be the superior method of entry as compared to typing. Copying and pasting a medication with the dosing is much safer compared to the use of a drop down menu since there are known errors related to wrong selections and jumping menus in the use of drop down menus. The 'copy and paste' function should always be associated with a clear "chain of custody". As a general rule, the use of 'copy and paste' should be discouraged in new orders to reinforce the fact of conscious data entry.

Medication entry when done in free text form, 'copy and paste' function would be considered the superior method when compared to typing. Copying and pasting the drugs could avoid spelling errors, dosage errors, frequency errors, etc. Copying and pasting a medication with the dosing is much safer compared to the drop down menu since the NIST research ${ }^{1}$ has shown errors related to wrong selection, jumping menu, etc. The 'copy and paste' function should always be associated with a clear chain of custody. Provisions should be made available to enable the user to copy the complete set of information to avoid any safety-related issues associated with incomplete documentation of information. In general, use of 'copy and paste' should be discouraged especially in new orders to reinforce the fact of conscious data entry.

\section{Discharge Summary}

When used during patient discharge, the 'copy and paste' of reused information should reflect the "chain of custody" for the clinical provider indicating that it is indeed copied from a past event.

A discharge summary is a good example of a location where 'copy and paste' functionality increases efficiency and efficacy, so long as the clinical provider is presented the "chain of custody" of such information. The initial presentation and the ultimate outcomes, the medications or treatments the patient was instructed to pursue could be easily and efficiently copied into the discharge summary but, the source of the copied information with a clear attribute to the original author needs to be associated with such information.

\section{Copying and Pasting Information from Different Departments and Another Patient's EHR}

When copying and pasting information into an EHR from a different patient's chart is allowed, the EHR system must keep the clinician oriented as to which patient's record they are accessing at any given point in the process. There should be a clear "chain of custody" indicating the exact source of the copied and pasted information while protecting the privacy and confidentiality of the source patient. Enable user to easily transition from the current chart with unrestricted access to input information to another chart by a deliberate action (i.e., identification / activation of the patient chart), by the user.

Special care must be taken when copying and pasting information into the EHR from a different patient's record. Our study revealed that at certain times, the efficiency is increased by allowing the 'copy and paste' function from one patient record to another. Copying information from a twin sibling's chart to the other's and from mother to the newborn baby to indicate the childbirth

${ }^{1}$ Lowry, S.Z., et. al. (2015). Technical Evaluation, Testing, and Validation of the Usability of Electronic Health Records: Empirically Based Use Cases for Validating Safety-Enhanced Usability and Guidelines for Standardization. NISTIR 78041 . 
information are some examples where the 'copy and paste' function increases the efficiency of the documentation process. If this capability is allowed, the EHR system must keep the clinician oriented as to which patient's record they are accessing at any given point in the process. There should be a clear "chain of custody" indicating the exact source of the copied and pasted information while protecting the privacy and confidentiality of the source patient. Enabling the user to easily transition from the current chart with unrestricted access to input information to another chart by a deliberate action (i.e., identification / activation of the patient chart), would help prevent errors of documentation in wrong patient chart. 


\begin{tabular}{|c|c|}
\hline \multicolumn{2}{|c|}{ Human Factors Recommendations to support The Partnership Recommendation A } \\
\hline $\begin{array}{l}\text { Provide a mechanism to make 'copy and } \\
\text { paste' material easily identifiable }\end{array}$ & $\begin{array}{l}\text { - EHR systems must be designed to enhance the } \\
\text { visibility of the information being selected for } \\
\text { 'copy and paste' to prevent users from } \\
\text { inadvertently copying only part of the information } \\
\text { that was intended to be copied which could } \\
\text { minimize the possibility of incomplete reuse of } \\
\text { information that could lead to morbid/mortal } \\
\text { errors. } \\
\text { EHR systems should provide a concept for } \\
\text { reconciling that the copied information was read } \\
\text { consciously and edited by the clinical provider } \\
\text { which would promote the attribution of the source } \\
\text { of the information. }\end{array}$ \\
\hline $\begin{array}{l}\text { Areas where 'copy and paste' should be } \\
\text { locked }\end{array}$ & $\begin{array}{l}\text { - The 'copy and paste' function must not be } \\
\text { allowed when entering any information into a } \\
\text { blood bank information system due to the extreme } \\
\text { risk involved in blood transfusion. } \\
\text { - Demographic information should never be } \\
\text { copied, but needs to be autopopulated in all the } \\
\text { interfaces within a patient's chart. } \\
\text { - Copying of demographic data from one chart to } \\
\text { antoher should NOT be allowed. } \\
\text { - Dates should never be copied and pasted. }\end{array}$ \\
\hline
\end{tabular}

\section{Human Factors Recommendation to support The Partnership Recommendation B}

Ensure that the provenance of 'copy and paste' material is readily available
- User interface must display the "chain of custody" of the information associated with the use of 'copy and paste'. However, this information should not be displayed by default, and only be shown on user demand to avoid the possibility of overwhelming clinical users and contribute to errors of commission (taking an incorrect action).

\section{Additional Human Factors Recommendations for critical areas identified in the study}

Vital signs
- $\quad$ The following properties should be displayed when documenting vital signs through charting done by the clinical user or by real time feed to the EHR system from digital devices:

$>$ Time stamp when measured. How it was measured (nurse manually takes BP, arterial line captures BP at a single time) 


\begin{tabular}{|c|c|}
\hline & $\begin{array}{l}>\text { Time stamp when documented in the EHR. } \\
>\text { Time stamp when it is signed (locked). } \\
>\text { Time stamp when it is revised. } \\
>\text { Time stamp when retrieved. }\end{array}$ \\
\hline Allergies & $\begin{array}{l}\text { - There should be clear "chain of custody" when } \\
\text { copying and pasting information related to } \\
\text { allergies. }\end{array}$ \\
\hline Surgical notes & $\begin{array}{l}\text { The design of the EHR must ensure that all of the } \\
\text { information pertaining to surgical notes is provided with } \\
\text { the 'copy and paste' functionality which helps the reuse } \\
\text { of information by the clinical staff in the "exact" and } \\
\text { precise context of the surgical notes. Variability may } \\
\text { exist due to patient-specific findings and/or specific } \\
\text { situations that might arise unexpectedly during the } \\
\text { procedure which must be accommodated by EHRs by } \\
\text { means of provisions for efficient editing. }\end{array}$ \\
\hline Medication entry & $\begin{array}{l}\text { - When medication entry is done in free text form, } \\
\text { the 'copy and paste' function could be the } \\
\text { superior method of entry as compared to typing. } \\
\text { Copying and pasting a medication with the } \\
\text { dosing is much safer compared to the use of a } \\
\text { drop down menu since there are known errors } \\
\text { related to wrong selections and jumping menus in } \\
\text { the use of drop down menus. } \\
\text { The 'copy and paste' function should always be } \\
\text { associated with a clear "chain of custody". As a } \\
\text { general rule, the use of 'copy and paste' should } \\
\text { be discouraged in new orders to reinforce the fact } \\
\text { of conscious data entry. }\end{array}$ \\
\hline Discharge summary & $\begin{array}{l}\text { When used during patient discharge, the 'copy and } \\
\text { paste' of reused information should reflect the "chain of } \\
\text { custody" for the clinical provider indicating that it is } \\
\text { indeed copied from a past event. }\end{array}$ \\
\hline $\begin{array}{l}\text { Copying and pasting information from } \\
\text { different departments and another } \\
\text { patient's EHR }\end{array}$ & $\begin{array}{l}\text { When copying and pasting information into an EHR from } \\
\text { a different patient's chart is allowed, the EHR system } \\
\text { must keep the clinician oriented as to which patient's } \\
\text { record they are accessing at any given point in the } \\
\text { process. There should be a clear "chain of custody" } \\
\text { indicating the exact source of the copied and pasted } \\
\text { information while protecting the privacy and } \\
\text { confidentiality of the source patient. Enable user to } \\
\text { easily transition from the current chart with unrestricted } \\
\text { access to input information to another chart by a } \\
\text { deliberate action (i.e., identification / activation of the } \\
\text { patient chart), by the user. }\end{array}$ \\
\hline
\end{tabular}

Table 7: Human Factors Conclusions of the 'Copy and Paste' Function 


\section{Conclusions}

This study explored the ways in which clinical providers (nurses and physicians) use EHRs in their work and specifically, if and how they use the 'copy and paste' function in their daily work using EHRs. The major findings of the usability study are:

1. Study participants expressed concerns about the integrity of information while using the 'copy and paste' function.

2. Study participants identified a high potential risk of entering wrong information in the wrong chart.

3. Study participants revealed critical issues regarding the 'copy and paste' function in producing "hyper-documentation" or "note bloat," which makes it difficult to extract accurate, relevant and timely information on a patient.

Data from this study overwhelmingly support Recommendation A and Recommendation B, of the ECRI convened Partnership for Health IT Patient Safety. There is no direct data to report on Recommendation $\mathrm{C}$ and Recommendation D, although there are some general indicators pointing toward ways in which the participants tangentially provided support for Recommendation $\mathrm{C}$ related to training.

In conclusion, the human factors recommendations provided above complement the ECRI Institute convened Partnership for Health IT Patient Safety's recommendations for safe use of 'copy and paste.' They add empirical evidence based human factors methodology to the safe use of 'copy and paste' emphasized by the Partnership recommendations.

Participants' general perception on the "copy and paste' function is that it is a "valuable tool" but, it should not be used "in all circumstances." The "copy and paste' function could save time and has the potential to be more reliable than relying on memory or users' typing skills. However, due to the way the 'copy and paste' function is used currently, there is tremendous potential for error and negative effects on patient safety.

The results of the study confirmed that 'the 'copy and paste' function must be improved by providing assurances for the integrity of the information that is copied and pasted. Series of the Human Factors recommendations on and guidance for the user interface usability are provided in this report.

The research uncovered additional challenges in the information/data transmitted from the medical devices. The study also pointed towards challenges associated with reuse of information in the form of autopopulation and information carry forward. The study revealed there are gaps in the knowledge about the impact of these functionalities and more research is needed. 


\section{Appendices}

\subsection{Appendix A: Demographic Questionnaire}

1. Please indicate your age range.

$\square 18-24$

$\square 25-34$

口 $35-44$

$45-54$

$55-64$

65 or older

2. Which one of the following educational background descriptions applies to you?

$\square$ Some high school

G.E.D. (General Education Development)

$\square$ High school diploma

$\square$ College degree

$\square$ Associate's degree

$\square$ Undergraduate degree; specify which one (e.g., BA, BS)

$\square$ Graduate degree; specify which one (e.g., MA, MS, PhD, MD, RN)

$\square$ Special certifications; specify which ones

Other; describe

3. What is your occupational title?

4. How many years have you worked as a [occupation title]?

5. What is your comfort level using computers in the workplace?

$\square$ Low: I am somewhat intimidated using computers

$\square$ Medium: I am relatively comfortable using computers

High: I am very comfortable using computers

6. What is your comfort level using computers at home?

$\square$ Low: I am somewhat intimidated using computers

$\square$ Medium: I am relatively comfortable using computers

High: I am very comfortable using computers

7. How frequently do you use computers outside of work?

$\square$ Regularly: Several times a week or more

$\square$ Rarely: Less than once a month

$\square$ Infrequently: Several times a month

8. How long have you have you been interacting with your current electronic health record system (what we will now refer to as an EHR)?

$\square$ Regularly: Several times a week or more

$\square$ Rarely: Less than once a month

$\square$ Infrequently: Several times a month 
9. Did you have any prior experience with other EHR systems before you started using your current system?

10. Describe the environment(s) in which you use your EHR (e.g., in the exam room with the patient, during rounds, in an office, at an open workstation in the unit, at home)?

11. On what kinds of device(s) do you interact with your EHR (e.g., workstation, workstation on wheels, laptop computer, PDA, smartphone)?

12. How do you interact with your EHR (e.g., touchscreen, mouse and keyboard, keypad, stylus, voice entry)? 


\subsection{Appendix B: Post-Task and Post-Test Questions}

\section{Post-Task Questions}

1. Please rate the task's ease of use (scale: $1=$ low, $7=$ high)

2. Where and how would you use "“copy and paste" " to optimize your record entry?

3. What EHR areas of record screen can be potential unsafe to be copied and pasted in?

4. Please rate the likelihood of committing a use error use (scale: 1=low, $7=$ high)

5. Do you recall committing any use errors, or times when you made a mistake while performing the task copying and pasting information?

6. Do you recall any "close calls," cases in which you almost committed a use error or did so but were immediately able to recover from the use error while copying and pasting?

7. Do you recall experiencing any "operational difficulties," cases in which you encountered obstacles to completing the task with the desired confidence, accuracy, precision or speed, for example?

8. What areas in the records, in your opinion, could be potentially unsafe to paste the information in?

9. What areas in the records, in your opinion, could be potentially safe and beneficial to paste information in?

\section{Post-Test Questions}

1. What is your overall impression of the complexity of the tasks you performed today using the system?

2. Where was 'copy and paste' functionality especially useful to complete your task in the EHR?

3. What suggestions do you have for making the EHR safer to use while copying and pasting information?

4. What suggestion do you have for making the EHR more efficient to use while copying and pasting information?

5. Which aspects of the system, if any, might lead users to commit use errors that could negatively affect patients' health? How could you improve the system to decrease the likelihood that clinicians might make use errors in relation to "“copy and paste"”?

6. Identify specific areas where in your opinion 'copy and paste' functionality should be available to the user, and the areas where this feature may not be safe, and therefore, should not be available to the users. 


\subsection{Appendix C: Semistructured In-depth Interview}

Note: This semi-structured interview document is meant as a discussion guide; it is not a script and therefore will not be read verbatim. The moderator will use these questions as a roadmap and probe as needed to maintain the natural flow of conversation.

\section{GENERAL INTERVIEW QUESTIONS}

1. Tell me a little bit about what you do in your current position?

- If they do not mention responsibilities, follow up by asking about responsibilities they have in their current position.

2. What word best describes your feelings about the use of your current EHR system?

- Why (insert participant's word)?

3. Generally speaking, how would you describe your experience using EHRs?

- If they do not mention likes and dislikes, follow up with a question about which aspects they like and/or dislike.

- If they do not mention what makes it easy and/or difficult to use, follow up with a question about ease and/or difficulty of use.

- If they do not mention positives and negatives, follow up with a question about what they see as positives and negatives in their experience using EHRs.

4. Can you describe functions or aspects of the EHR system you currently use that you think could lead to safety issues?

- What type of safety issues could arise related to this function?

- Can you think of things that might make this function safer?

5. On a scale of $1-5$, with 1 being "not at all difficult" and 5 being "extremely difficult," how difficult was this task?

Not At All Slightly

1

Moderately

3

Very

4

Extremely

5

6. What made the task (insert participant's rating)?

7. On a scale of $1-5$, with 1 being "not very likely" and 5 being "extremely likely," how likely do you think it would be for someone to commit a use error while doing this task?

Not At All Slightly Moderately Very $\quad$ Extremely

$\begin{array}{llllll}1 & 2 & 3 & 4 & 5\end{array}$

8. How would you describe your experience using the EHR today?

- If they do not mention what was easy and/or difficult, follow up with a question about ease and/or difficulty of accomplishing the tasks. 
9. Can you describe any particular times during the scenario when you felt at risk of making a mistake or a use error?

- Probe for specifics here, about what was going on in the task and their use of the EHR.

10. Can you describe what might have contributed to use errors or mistakes while completing the task?

- Probe to see if there were particular functions, areas in records, or operational issues for example that contributed to use errors or mistakes.

11. Were there any particular functions or tools that you believe could lead to safety issues when trying to accomplish tasks like these?

Possible probes, if needed

- What types of safety issues to you think could have occurred?

- What might have contributed to safety issues?

- How do you think these safety issues might be avoided?

12. What do you think are the most important things someone needs to think about when using an EHR?

Possible probes if needed

- Are there any official best practices or standards of use that you are aware of related to EHR systems?

O If they do not know of any best practices, ask: Do you think there should be best practices related to the use of EHR systems? Why or why not?

- Have you developed your own set of "best practices" related to how you use EHRs?

- Which of these practices do you feel are most essential to adhere to when using an EHR system.

- If you were designing a set of best practices for using EHRs, what would you include? Why these things?

13. Can you describe any safety issues you believe are related to the ways in which EHRs are used today?

- Are there specific functions or tools in EHRs that contribute to these?

14. If you could design your own EHR system, what functions would you make sure to include.

- Why these?

15. And what functions would you make sure were NOT included?

- Why?

16. Can you describe if and how you use the 'copy and paste' Function with your current EHR system?

- In what ways, if at all, is this function helpful?

- What types of problems, if any, do you think might happen when using this function?

- Are there particular areas in a record where you think it is particularly dangerous to use the 'copy and paste' function? 
○ If so, what are they and why do you feel they are particularly unsafe?

- Are there particular areas in a record where you believe it is potentially safe to use the 'copy and paste' function?

- If so, what are they and why do you feel they are potentially safe?

- Can you describe particular features or user interfaces, if any, that you believe might make the 'copy and paste' function safer to use?

- Are there particular ways in which you think pasted information should be displayed on the screen?

- Can you describe some of these and why/how you think this will be helpful? 


\subsection{Appendix D: Testing Scenarios and Tasks}

\section{Test Scenario and Tasks for Surgeon}

\section{Your role today}

You are a surgeon, Dr. Echo Doctor and in the $200 \mathrm{H}$ Clinic today.

\section{Overview}

Mr. Dean L. Howe is a 40-year-old male who underwent a right above-the-knee amputation by you this morning. He is being transferred to the rehabilitation center due to lack of availability of beds in the $200 \mathrm{H}$ Clinic.

For the transfer, you need to enter a clinical note containing the patient identification information and the entire surgical notes.

\section{Task 1: Access and review/study Dean L. Howe's chart}

Mr. Dean L. Howe is a 40-year-old male who underwent a right above-the-knee amputation by you this morning.

Open Dean L. Howe's chart and review/study the surgical encounter.

1. Open "Appointments."

2. Open the chart of Dean L. Howe.

3. Review the surgical details from the "Current Encounter."

\section{Task 2. Enter Dean L. Howe's scenario specific information}

He is being transferred to the rehabilitation center due to lack of availability of beds in the $200 \mathrm{H}$ Clinic.

For the transfer, you need to enter a clinical note containing the patient identification information and the entire surgical notes.

Create a clinical note for Dean L. Howe and enter the patient identification information and the entire surgical notes from today.

1. Open the "Clinical note."

2. Enter the patient identification information.

3. Refer to the surgical notes in today's encounter by clicking the edit note button next to the surgical notes seen.

4. Enter the entire surgical notes from today into the clinical note.

5. Save.

6. Exit. 


\section{Test Scenario and Tasks for Primary Care Physician}

\section{Your role today}

You are a primary care physician Dr. Charlie Doctor and in the $200 \mathrm{H}$ Clinic today.

\section{Overview}

Your long time patient, 68-year-old Jessica B. Barber is with you today. She has had problems with her Medicare reimbursement for her visit to the PCP's Emergency Clinic on 19 Feb 2014. She has a mail note from Medicare demanding her to request her PCP (that is you) to email them the encounter details of this visit. Otherwise, she will end up paying $\$ 2000$ out of pocket.

They require you to have the following information at the beginning of the email.

Attention:

Medicare Claims Department

Patient Name:

Date of Birth:

Record Number:

Subject: Encounter details of Jessica B. Barber for her visit to Emergency Care Clinic on February 19, 2014.

Task 1: Access and review/study the chart of Jessica B. Barber.

Your long time patient, 68-year-old Jessica B. Barber with you today. She has had problems with her Medicare reimbursement for her visit to the PCP's Emergency Clinic on 19 Feb 2014. She has a mail note from Medicare demanding her to request her PCP (that is you) to email them the encounter details of this visit. Otherwise, she will end up paying $\$ 2000$ out of pocket.

Open Jessica B. Barber's chart and review/study the chart.

1. Open "Appointments".

2. Open the chart of "Jessica B. Barber."

3. Review the patient details from the information in "Previous encounters" pertaining to this scenario.

4. Save.

\section{Task 2: Enter Jessica Barber's scenario specific information}

Jessica Barber has had problems with her Medicare reimbursement for her visit to the PCP's Emergency Clinic on 19 Feb 2014. She has a mail note from Medicare demanding her to request her PCP (that is you) to email them the encounter details of this visit. Otherwise, she will end up paying $\$ 2000$ out of pocket. They require you to have the following information at the beginning of the email. Attention:

Medicare Claims Department

Patient Name:

Date of Birth:

Record Number:

Subject: Encounter details of Jessica B. Barber for her visit to Emergency Care Clinic on February 19, 2014.

Create a clinical note and write a note addressing the Medicare Claims Department with all the information from the encounter dated Feb. 19, 2014.

1. Open the "Clinical note" within Jessica B. Barber's chart.

2. Enter the information that Medicare requires in the beginning of the email.

3. Enter all the Feb. 19, 2014 encounter details.

4. Save to be emailed later.

5. Exit. 


\section{Your role today}

\section{Test Scenario and Tasks for Pediatric Critical Care Physician}

You are a pediatric critical care physician Dr. Echo Doctor and in the $200 \mathrm{H}$ Clinic today.

\section{Overview}

You are attending to 2 critical children who presented to the ER after an accident at a local amusement park. Parents indicate that both 9-year-old Odette D. Barnett and 9-year-old Lawrence E. Carpenter fell from adjacent slide boards which gave way suddenly from approximately 20 feet from the ground and landed onto the concrete floor with an impact. The children are complaining that their heads and chests "hurt." You suspect head and chest injuries as a result of the fall. You have done the preliminary investigations and initiated the treatment. Both children will be transferred to the head trauma center. You have completed the transfer note for Lawrence E. Carpenter following the format your practice requires. Now, you have examined and treated Odette and are documenting the transfer note (which needs to be documented as a clinical note) for Odette in the same format and content.

\section{Patient Odette D. Barnett}

Odette D. Barnett is with you today accompanied by her parents. She fell from adjacent slide boards with her friend Lawrence which gave way suddenly from approximately 20 feet from the ground and landed onto the concrete floor with an impact. She complains that her head and chest "hurts." Odette was conscious on presentation at the clinic and complained of worsening headache, hazy vision, 4 episodes of vomiting and loss of consciousness for half an hour after the fall. Odette is a known to have Hepatitis B.

Examination revealed abnormal pupillary reflexes, decreased level of consciousness and lateralizing features. Vital signs at presentation BP - 120/40 HR - $40 \mathrm{RR}-15$ and irregular Temp. 98.4 deg. F HT -53 inches WT $-55 \mathrm{lbs}$.

CT shows subdural hematoma with increased intracranial pressure. Chest X-ray shows fracture of ribs 4,5 , and 6 on the right side without signs of pneumothorax.

Odette was diagnosed with posttraumatic subdural hematoma. Cushing's triad was identified at presentation, suggestive of raised intracranial pressure.

Odette was offered the same first line of treatment that Lawrence E. Carpenter was given. She was intubated with a $6 \mathrm{~mm}$ endotracheal tube secured at $16 \mathrm{~cm}$ at the lip.

Odette is now being transferred to the head trauma center for further management. Her Vital signs just before transfer are BP - 100/80 HR - 98 Temp. 98.4 deg. F.

You document the transfer note (which needs to be documented in a "Clinical Note") for Odette in the same format and content as Lawrence E. Carpenter per your practice requirements.

\section{Task 1: Access and identify Odette D. Barnett's chart}

Odette D. Barnett is with you today accompanied by her parents.

Open Odette D. Barnett's chart and identify the chart with the information available.

1. Open "Appointments".

2. Open the chart of "Odette D. Barnett."

3. Confirm the patient chart with the available information.

\section{Task 2: Enter Odette Barnett's reason for today's visit, history of present illness and the vital signs.}

Odette D. Barnett is with you today accompanied by her parents. She fell from adjacent slide boards with her friend Lawrence which gave way suddenly from approximately 20 feet from the ground and landed onto the concrete floor with an impact. She complains that her head and chest "hurts." Odette was conscious on presentation at the clinic and complained of worsening headache, hazy vision, 4 
episodes of vomiting and loss of consciousness for half an hour after the fall. Odette is a known to have Hepatitis B.

Examination revealed abnormal pupillary reflexes, decreased level of consciousness and lateralizing features. Vital signs at presentation are BP - 120/40 HR - $40 \mathrm{RR}-15$ and irregular Temp. 98.4 deg. F HT - 53 inches WT - 55 lbs.

1. Open the "Clinical note" within Odette D. Barnett's chart.

2. Enter patient identification information (Name, MRN, Date of Birth)

3. Enter the reason for today's visit.

4. Enter history of present illness (HPI).

5. Enter the vital signs.

6. Save.

\section{Task 3: Enter Odette D. Barnett's scenario specific information}

CT shows subdural hematoma with increased intracranial pressure. Chest X-ray shows fracture of ribs 4,5 , and 6 on the right side without signs of pneumothorax.

Odette was diagnosed with posttraumatic subdural hematoma. Cushing's triad was identified at presentation, suggestive of raised intracranial pressure.

Odette was offered the same first line of treatment that Lawrence E. Carpenter was given. She was intubated with a $6 \mathrm{~mm}$ endotracheal tube secured at $16 \mathrm{~cm}$ at the lip.

Odette is now being transferred to the head trauma center for further management. Her Vital signs just before transfer are BP - 100/80 HR - 98 Temp. 98.4 deg. F.

You document the transfer note (which needs to be documented in a "Clinical Note") for Odette in the same format and content as Lawrence E. Carpenter per your practice requirements.

Enter assessment and plan with the treatment given and the intention to transfer for further care.

1. Enter all the neurological examination findings.

2. Enter the findings from the radiological reports.

3. Enter the impression.

4. Enter the treatment information.

5. Enter all the concluding information just before transfer.

6. Save.

7. Exit. 


\section{Your role today}

\section{Test Scenario and Tasks for Pediatrician}

You are a pediatrician, Dr. Alpha Doctor and in the $200 \mathrm{H}$ Clinic today.

Your group practice, since the last month, requires a detailed description of the administration of every vaccine to be recorded in the Clinical notes. This description has to include the name of the vaccine, route of administration, any reaction that may occur during the administration and instructions to the patient. There is no standard template available for the typical vaccine administration notes. But, this morning, you had created and entered a clinical note with the typical vaccine administration notes for a patient, Tatiana Jarvis, during her visit to you for the administration of the Varicella vaccine.

\section{Overview}

Mrs. Kimberly Coffey is visiting your clinic today with her three sons, Casey, 9 years old, Brody, 5 years old and Kane, 4 years old. She is here for the Varicella vaccine to be administered to Brody and Kane. She also requests for a concise clinical note for Casey mentioning all the immunizations he has received, so far, and the dates they were administered. She needs this for documentation during Casey's upcoming international travel.

\section{Task 1: Patient Brody Coffey}

Brody Coffey is a 5-year-old male child who is here today accompanied by his mother. He is here for his Varicella vaccination. He weighs $50 \mathrm{lbs}$. and is 48 inches tall. His heart rate is 108, respiratory rate 23, and temperature $98.4 \mathrm{deg}$. F. Mother carries a record of the patient when the first dose of Varivax was administered at the age of 15 months. You have now administered Brody Coffey with Varivax injection. This is his second dose. There were no complications noted. The quantity, lot number and the route of administration have remained the same as for Tatania Jarvis.

Please enter a new "Clinical note" in detail for Brody Coffey following the steps below.

\section{Task 1.1: Access and review/study Brody Coffey's chart}

Brody Coffey is a 5-year-old male child who is here today accompanied by his mother.

Open Brody Coffey's chart and review/study the chart.

1. Open "Appointments".

2. Open the chart of "Brody Coffey."

3. Review the patient details from "Health History" or "Previous encounters."

\section{Task 1.2: Enter Brody Coffey's reason for today's visit, history of present illness and the vital signs.}

Brody Coffey is a 5-year-old male child who is here today accompanied by his mother. He is here for his Varicella vaccination. He weighs $50 \mathrm{lbs}$. and is 48 inches tall. His heart rate is 108, respiratory rate 23, and temperature 98.4. Mother carries a record of the patient when the first dose of Varivax was administered at the age of 15 months.

Enter the reason for today's visit, history of present illness and the vital signs.

1. Open a new "Clinical Note."

2. Enter the reason for appointment.

3. Enter the vital signs.

Task 1.3: Enter Brody Coffey's scenario specific information. 
You have now administered Brody Coffey with Varivax injection. This is his second dose. There were no complications noted. The quantity, lot number and the route of administration have remained the same as for Tatania Jarvis.

Enter vaccine administration notes in the chart of Brody Coffey. Please note that your practice requires a detailed vaccination notes as mentioned above.

1. Enter the vaccination information similar to that of the patient Tatania Jarvis.

2. Exit.

\section{Task 2. Patient Kane Coffey}

Kane Coffey is a 4-year-old male child who is here today, accompanied by his mother. He is here for his Varicella vaccination. He weighs $34 \mathrm{lbs}$. and is 37 inches tall. His heart rate is 99, respiratory rate 26, and temperature 99. Mother carries a record of the patient when the first dose of Varivax was administered at the age of 15 months.

You have now administered Kane Coffey with $0.5 \mathrm{ml}$ of Varivax subcutaneously. This is his second dose. Kane felt lightheaded after the vaccine administration for which he was observed for half an hour. No other complications noted.

Please enter the Clinical notes in detail for Kane Coffey following the steps below.

\section{Task 2.1: Access and review/study Kane Coffey's chart}

Kane Coffey is a 4-year-old male child who is today, accompanied by his mother.

Open Kane Coffey's chart and review/study the chart.

1. Open "Appointments".

2. Open the chart of "Brody Coffey."

3. Review the patient details from "Health History" or "Previous encounters."

\section{Task 2.2: Enter Kane Coffey's reason for today's visit and the vital signs.}

Kane Coffey is a 4-year-old male child who is today, accompanied by his mother. He is here for his Varicella vaccination. He weighs $34 \mathrm{lbs}$. and is 37 inches tall. His heart rate is 99, respiratory rate 26, and temperature 99. Mother carries a record of the patient when the first dose of Varivax was administered at the age of 15 months.

1. Open a new "Clinical Note."

2. Enter the reason for appointment.

3. Enter the history of present illness (HPI).

4. Enter the vital signs.

\section{Task 2.3: Enter Kane Coffey's scenario specific information}

You have now administered Kane Coffey with $0.5 \mathrm{ml}$ of Varivax subcutaneously. This is his second dose. Kane felt lightheaded after the vaccine administration for which he was observed for half an hour. No other complications noted. The quantity, lot number and the route of administration have remained the same as for Tatania Jarvis.

Enter vaccine administration notes in the chart of Kane Coffey. Please note that your practice requires a detailed vaccination notes as mentioned above.

1. Enter the vaccination information similar to that of the patient Tatania Jarvis. Also, add appropriately that Kane had lightheadedness after vaccination for which he was under observation for half an hour.

2. Save. 


\section{Exit.}

\section{Task 3. Patient Casey Coffey}

Casey Coffey is a 9-year-old male child who is here today, accompanied by his mother. The mother requests for a concise clinical note for Casey, mentioning all the immunizations he has received, so far, and the dates they were administered. She needs this for documentation during Casey's upcoming international travel. He weighs $90 \mathrm{lbs}$. and is 55 inches tall. His heart rate is 96, respiratory rate 20, and temperature 98.2.

You thoroughly review Casey's chart and the notes in the previous encounters; makes a note of all vaccines administered to Casey; and enters it to the clinical notes.

\section{Task 3.1: Access and review/study Casey Coffey's chart}

Casey Coffey is a 9-year-old male child who is here today, accompanied by his mother.

Open Casey Coffey's chart and review/study the chart.

5. Open "Appointments."

6. Open the chart of Casey Coffey.

7. Review the patient details from "Health History" and "Previous encounters."

\section{Task 3.2: Enter Casey Coffey's reason for today's visit and the vital signs.}

Casey Coffey is a 9-year-old male child who is here today, accompanied by his mother. The mother requests for a concise clinical note for Casey, mentioning all the immunizations he has received, so far, and the dates they were administered. She needs this for documentation during Casey's upcoming international travel. He weighs $90 \mathrm{lbs}$. and is 55 inches tall. His heart rate is 96, respiratory rate 20, and temperature 98.2.

Create a clinical note for Casey Coffey and enter the reason for today's visit and the vital signs.

7. Open a new "Clinical note."

8. Enter the reason for visit and the vital signs.

\section{Task 3.3: Enter Casey Coffey's scenario specific information}

Casey Coffey's mother requests for a concise clinical note for Casey, mentioning all the immunizations he has received, so far, and the dates they were administered. She needs this for documentation during Casey's upcoming international travel. You thoroughly review Casey's chart and the notes in the previous encounters; makes a note of all vaccines administered to Casey; and enters it to the clinical notes.

Enter the information of all the vaccines that Casey Coffey has received so far and also the corresponding dates of administration.

1. Enter all the vaccination information requested.

2. Save.

3. Exit. 


\section{Test Scenario and Tasks for Intensive Care Nurse}

\section{Your role today}

You are a nurse by name Dave Nurse in the Emergency Room of the $200 \mathrm{H}$ Clinic.

\section{Overview}

You are attending patient 71-year-old male patient Wayne T. Cabrera. The ER physician has asked you for a note in the current encounter specifying the findings of the CT report in the clinical note dated July 18, 2016 at 0138 since he is not able to access them from his system. He also requests her to enter the patient identification information at the beginning of the note.

\section{Tasks}

\section{Task 1. Access and review/study Wayne T. Carbrera's chart}

You are attending patient 71-year-old male patient Wayne T. Cabrera.

Open Wayne T. Cabrera's chart and review/study the chart.

1. Open "Appointments".

2. Open the chart of "Wayne T. Cabrera."

3. Open "Previous encounters."

4. Review the patient information.

The ER physician has asked you for a note in the current encounter specifying the findings of the CT report in the clinical note dated July 18, 2016 at 0138 since he is not able to access them from his system. He also requests her to enter the patient identification information at the beginning of the note.

\section{Task 2. Enter Wayne T. Carbrera's scenario specific information}

B. Enter the patient identification information into the "AddNote" in the "Current Encounter" at the beginning. Then enter the findings seen in the CT report of July 18, 2016 at 0138 clinical note.

1. Enter the patient identification information.

2. Enter the CT report findings.

3. Save. 


\section{Test Scenario and Tasks for Pediatric Nurse}

\section{Your role today}

You are a nurse by name Cathy Nurse in the Pediatric Clinic.

\section{Overview}

Sopoline L. Burch, a 4-year-old child is in the clinic for referral to the growth clinic. She has only a 2inch increase in height since her last visit in Sep. 2, 2014. Today's vital signs have remained same as the ones in the previous encounter dated Sep. 2, 2014 except the height is 2 inches more. The attending pediatrician has requested you to pull the growth chart information displayed below the growth chart starting Oct. 2012 and enter it onto the current encounter which could be used for referral to the growth clinic.

Tasks

\section{Task 1. Access and review/study Sopoline L. Burch's chart}

Sopoline L. Burch, a 4-year-old child is in the clinic for referral to the growth clinic.

Open Sopoline L. Burch's chart and review/study the chart.

1. Open "Appointments".

2. Open the chart of "Sopoline L. Burch."

3. Open "Previous encounters."

4. Review the patient information.

\section{Task 2. Enter Sopoline L. Burch's the vital signs.}

She has only a 2-inch increase in height since her last visit in Sep. 2, 2014. Today's vital signs have remained same as the ones in the previous encounter dated Sep. 2, 2014 except the height which is 2 inches more.

Enter today's vital signs data (has remained same as the ones in the previous encounter dated Sep. 2, 2014. You also specifically enter the growth chart data requested.

7. Open a Current Encounter.

8. Enter the reason for the visit.

9. Enter the vital signs.

\section{Task 3. Enter Sopoline L. Burch's scenario specific information}

The attending pediatrician has requested you to pull the growth chart information displayed below the growth chart starting Oct. 2012 till today and enter it onto the current encounter which could be used for referral to the growth clinic.

Enter the requested growth chart data

1. Get the requested growth chart data.

2. Enter in the current encounter.

3. Exit. 


\section{Test Scenario and Tasks for Peri-operative Nurse}

\section{Your role today}

You are perioperative nurse by name Susan Nurse and today, in a busy ambulatory surgery center located within the Clinic $200 \mathrm{H}$.

\section{Overview}

There are patients waiting for pre-op. You, who work there, has been delayed by traffic and now rushes and see patients waiting.

You have three patients 56-year-old male Nero G. Jimenez, 57-year-old female Charity N. Todd and 61-year-old male Blaze B. Fitzgerald waiting in pre-op. Each patient is waiting for a cholecystectomy. You do a quick physical exam on all three patients. Each one is unremarkable and within normal limits. You enter this in the text field of clinical note along with the patient identification information at the top of the first patient followed by the second and third patients, as well.

\section{Tasks}

\section{Task 1: Patient Nero G. Jimenez}

You have 56-year-old male patient Nero G. Jimenez for cholecystectomy pre-op. You have done a quick physical exam and now, start the process of documentation.

You recorded the patient's vital signs today; BP: 114/75, HR: 82, RR: 18, T: 99.2 deg. F, HT: 69 inches, WT: 147 lbs., Tobacco Use: None, Alcohol Use: Binge. You document this information. You also need to enter the patient identification information available on the top of the chart in the beginning of the clinical note.

\section{Task 1.1: Access and review/study Nero G. Jimenez's chart}

You have 56-year-old male patient Nero G. Jimenez for cholecystectomy pre-op. You have done a quick physical exam and now, start the process of documentation.

Open Nero G. Jimenez's chart and match the chart with the patient.

4. Open "Appointments".

5. Open the chart of "Nero G. Jimenez."

6. Confirm the identifiers with the available information."

\section{Task 1.2: Enter Nero G. Jimenez's reason for today's visit and vital signs.}

You recorded the patient's vital signs today; BP: 114/75, HR: 82, RR: 18, T: 99.2 deg. F, HT: 69 inches, WT: 147 lbs., Tobacco Use: None, Alcohol Use: Binge. You document this information. You also need to enter the patient identification information available on the top of the chart in the beginning of the clinical note. 
Enter the patient identification information and the vital signs data you recorded today.

10. Open the clinical note in Nero G. Jimenez's chart.

11. Enter patient identification information.

12. Enter the vital signs.

13. Save.

14. Exit.

\section{Task 2. Patient Charity N. Todd}

You have 57-year-old female patient Charity N. Todd for cholecystectomy pre-op. You have done a quick physical exam and start the process of documentation.

You recorded the patient's vital signs today; BP: 108/73, HR: 90, RR: 17, T: 98.8 deg. F, HT: 65 inches, WT: 112 lbs., Tobacco Use: None, Alcohol Use: Low. You document this information. You also need to enter the patient identification information available on the top of the chart in the beginning of the clinical note.

\section{Task 2.1: Access and review/study Charity N. Todd's chart}

You have 57-year-old female patient Charity N. Todd for cholecystectomy pre-op. You have done a quick physical exam and start the process of documentation.

Open Charity N. Todd's chart and match the chart with the patient.

1. Open "Appointments".

2. Open the chart of "Charity N. Todd."

3. Confirm the identifiers with the available information.

\section{Task 2.2: Enter Charity N. Todd's reason for today's visit and vital signs.}

You recorded the patient's vital signs today; BP: 108/73, HR: 90, RR: 17, T: 98.8 deg. F, HT: 65 inches, WT: 112 lbs., Tobacco Use: None, Alcohol Use: Low. You document this information. You also need to enter the patient identification information available on the top of the chart in the beginning of the clinical note.

Enter the patient identification information and the vital signs data you recorded today.

1. Open the clinical note in Charity N. Todd's chart.

2. Enter patient identification information.

3. Enter the vital signs.

4. Save.

5. Exit.

\section{Task 3. Patient Blaze B. Fitzgerald}

You have 61-year-old male patient Blaze B. Fitzgerald for cholecystectomy pre-op. You do a quick physical exam and start the process of documentation.

\section{Task 3.1: Access and review/study Blaze B. Fitzgerald's chart}


A. Open Blaze B. Fitzgerald's chart and match the chart with the patient.

1. Open "Appointments".

2. Open the chart of "Blaze B. Fitzgerald."

3. Confirm the identifiers with the available information."

\section{Task 3.2: Enter Blaze B. Fitzgerald's reason for today's visit and vital signs.}

You recorded the patient's vital signs today; BP: 115/77, HR: 86, RR: 18, T: 99 deg. F, HT: 70 inches, WT: 112 lbs., Tobacco Use: None, Alcohol Use: None. You document this information. You also need to enter the patient identification information available on the top of the chart in the beginning of the clinical note.

Enter the patient identification information and the vital signs data you recorded today.

1. Open the clinical note in Blaze B. Fitzgerald's chart.

2. Enter patient identification information.

3. Enter the vital signs.

4. Save.

5. Exit. 


\section{Test Scenario and Tasks for Intensive Care Nurse}

\section{Your role today}

You are an Intensive Care Nurse by name Cathy Nurse in the $200 \mathrm{H}$ Clinic today.

\section{Overview}

You are attending to a 58-year-old male patient Mr. Amal L. Jefferson who is ready for his exam by the physician for a renal scan. You recorded the patient's vital signs today. He has the same vital sign recordings as noted in the encounter dated Sep. 12, 2014. So, you hurriedly enter the vital signs from today and his medication history from yesterday's primary physician's notes into today's visit. (The PCP has a special note in the bottom of today's encounter that Cipro was stopped effective that day.) You asked him if anything in his medications has changed to which he replied "no." Your medication history will be reconciled by the physician during the exam before the renal scan data entry.

\section{Tasks}

\section{Task 1. Access and identify Amal L. Jefferson's chart.}

You are attending to a 58-year-old male patient Mr. Amal L. Jefferson who is ready for his exam by the physician for a renal scan.

Open Amal L. Jefferson's chart and review/study the clinical note sent by the patient's PCP.

1. Open "Appointments".

2. Open the chart of "Amal L. Jefferson."

3. Identify the chart with the patient information available.

4. Review the patient information sent by the PCP which is the previous encounter dated July 19, 2016.

\section{Task 2: Enter Amal L. Jefferson's reason for today's visit and the vital signs.}

You are attending to a 58-year-old male patient Mr. Amal L. Jefferson who is ready for his exam by the physician for a renal scan. You recorded the patient's vital signs today. He has the same vital sign recordings as noted in the encounter dated Sep. 12, 2014. You enter these vital signs into his "Current Encounter."

Enter the vital signs data you recorded today.

1. Enter the chief complaint/reason for the visit.

2. Refer the previous encounter dated Sep. 12, 2014.

3. Enter the vital signs.

\section{Task 3. Enter Amal L. Jefferson's scenario specific information.}

So, you hurriedly enter the vital signs from today and his medication history from yesterday's primary physician's notes into today's visit. (The PCP has a special note in the bottom of today's encounter that Cipro was stopped effective that day.) You asked him if anything in his medications has changed to 
which he replied "no." Your medication history will be reconciled by the physician during the exam before the renal scan data entry.

Enter the medication history.

1. Refer to the clinical note dated July 19, 2016.

2. Enter the medication history provided by the patient's PCP in "AddNote" in the "Current Encounter." 


\section{Test Scenario and Tasks for General Medical-Surgical Nurse}

\section{Your role today}

You are a nurse by name Anna Nurse and in the pacemaker clinic within the $200 \mathrm{H}$ Clinic today.

\section{Overview}

You are checking 72-year-old male patient Colt O. Barber who is in the pacemaker clinic with an episode of syncopal attack an hour ago. Coincidentally, the vital signs you recorded today are exactly the same as seen in the previous encounter dated Nov. 14, 2011. While entering his screening information, you check for his pacemaker lead wire recall. You find the lead wire identification number from his clinical note dated July 19, 2016, which is a special note mentioning the lead wire number. You verify with the recall number list that the lead wire implanted in the patient Colt O. Barber has no recall. You enter into Mr. Barber's current encounter documentation by leaving a note (using AddNote) mentioning "all" the wire details mentioned in the July 19, 2016 clinical note and that it is not a part of the recall.

\section{Tasks}

You are checking 72-year-old male patient Colt O. Barber who is in the pacemaker clinic with an episode of syncopal attack an hour ago.

\section{Task 1: Access and review/study Colt O. Barber's chart}

Open Colt O. Barber's chart and review/study the patient past history.

1. Open "Appointments".

2. Open the chart of "Colt O. Barber."

3. Identify the chart with the information available.

4. Review the patient pacemaker lead wire information in the clinical note dated July 19, 2016.

\section{Task 2: Enter Colt O. Barber's reason for today's visit and vital signs}

You are checking 72-year-old male patient Colt O. Barber who is in the pacemaker clinic with an episode of syncopal attack an hour ago. Coincidentally, the vital signs you recorded today are exactly the same as seen in the previous encounter dated Nov. 14, 2011.

Enter the reason for visit, vital signs data and the lead wire recall information.

1. Open "Current Encounter"

2. Enter the reason for the visit.

3. Enter the vital signs.

\section{Task 3: Enter Colt O. Barber's scenario specific information}

While entering his screening information, you check for his pacemaker lead wire recall. You find the lead wire identification number from his clinical note dated July 19, 2016, which is a special note mentioning the lead wire number. You verify with the recall number list that the lead wire implanted in 
the patient Colt O. Barber has no recall. You enter into Mr. Barber's current encounter documentation by leaving a note (using AddNote) mentioning "all” the wire details mentioned in the July 19, 2016 clinical note and that it is not a part of the recall.

Enter the lead wire recall information.

1. Refer to the "Clinical Notes" with the lead wire information.

2. Open "AddNote" in the "Current Encounter."

3. Enter "all" the lead wire recall information with the wire details.

4. Exit. 